\title{
Multiloop amplitudes of light-cone gauge NSR string field theory in noncritical dimensions
}

\author{
Nobuyuki Ishibashi ${ }^{a}$ and Koichi Murakami ${ }^{b}$ \\ ${ }^{a}$ Center for Integrated Research in Fundamental Science and Engineering (CiRfSE), \\ Faculty of Pure and Applied Sciences, University of Tsukuba, \\ Tsukuba, Ibaraki 305-8571, Japan \\ ${ }^{b}$ National Institute of Technology, Kushiro College, \\ Otanoshike-Nishi 2-32-1, Kushiro, Hokkaido 084-0916, Japan \\ E-mail: ishibash@het.ph.tsukuba.ac, koichi@kushiro-ct.ac.jp
}

ABSTRACT: Feynman amplitudes of light-cone gauge superstring field theory are ill-defined because of various divergences. In a previous paper, one of the authors showed that taking the worldsheet theory to be the one in a linear dilaton background $\Phi=-i Q X^{1}$ with Feynman $i \varepsilon(\varepsilon>0)$ and $Q^{2}>10$ yields finite amplitudes. In this paper, we apply this worldsheet theory to dimensional regularization of the light-cone gauge NSR superstring field theory. We concentrate on the amplitudes for even spin structure with external lines in the (NS,NS) sector. We show that the multiloop amplitudes are indeed regularized in our scheme and that they coincide with the results in the first-quantized formalism through the analytic continuation $Q \rightarrow 0$.

Keywords: String Field Theory, BRST Quantization, Conformal Field Models in String Theory, Superstrings and Heterotic Strings

ARXIV EPRINT: 1611.06340 


\section{Contents}

1 Introduction 1

2 Superstring field theory in linear dilaton background $\quad 2$

2.1 Linear dilaton background 3

2.2 Light-cone gauge superstring field theory in linear dilaton background 5

2.3 Feynman amplitudes of light-cone gauge superstring field theory in linear dilaton background

$\begin{array}{lll}3 & \text { BRST invariant form of the amplitudes } & 9\end{array}$

4 The amplitudes from the first-quantized formalism 13

$\begin{array}{lll}4.1 & \text { The prescription } & 13\end{array}$

$\begin{array}{ll}4.2 Q \rightarrow 0 \text { limit of the light-cone gauge amplitudes } & 15\end{array}$

5 Conclusions and discussions $\quad 16$

$\begin{array}{ll}\text { A Arakelov metric and Arakelov Green's function } & 17\end{array}$

$\begin{array}{ll}\text { B Supersymmetric } X^{ \pm} \text {CFT } & 18\end{array}$

$\begin{array}{ll}\text { C Ghost systems on higher genus Riemann surfaces } & 21\end{array}$

$\begin{array}{ll}\text { C.1 A formula for the superghosts } & 23\end{array}$

$\begin{array}{lll}\text { C.2 A formula for the reparametrization ghosts } 26 & 26\end{array}$

$\begin{array}{ll}\text { D A proof of (3.9) } & 28\end{array}$

\section{Introduction}

Since the light-cone gauge superstring field theory [1-6] takes a simple form, this theory is expected to be very useful in studying superstring theory. In a series of papers [714], using the light-cone gauge closed NSR superstring field theory, we have studied the contact term divergences [15-19]. We have pointed out that the contact term divergences can be regularized by shifting the central charge of the light-cone gauge worldsheet theory to a sufficiently large negative value [7]. We refer to this type of regularization as the dimensional regularization, since the central charge is directly related to the spacetime dimensions in string theory. We have considered mainly two ways to shift the central charge in the regularization: the one is to naively shift the number of the transverse dimensions $d-2$; the other is to add a superconformal field theory with central charge large negative to the worldsheet theory. 
Recently, one of the authors has proposed another prescription [20], in which the string theory in a linear dilaton background $\Phi=-i Q X^{1}$ is considered so that the central charge of the system becomes $12-12 Q^{2}$. In [20], the divergences which appear in the amplitudes of this theory have been thoroughly studied. It has been shown that the Feynman amplitudes of light-cone gauge superstring field theory in the linear dilaton background are indeed finite, when the theory is with the Feynman $i \varepsilon(\varepsilon>0)$ and $Q^{2}>10$.

In this paper, we use this theory to dimensionally regularize and calculate the Feynman amplitudes. In this work, we restrict ourselves to the amplitudes for even spin structure with external lines in the (NS,NS) sector for simplicity. In this scheme, we define the amplitudes as analytic functions of $Q$ and perform the analytic continuation $Q \rightarrow 0$. We show that the limit $Q \rightarrow 0$ is smooth except the divergences coming from the boundaries of the moduli space and the results coincide with those from the first-quantized method. ${ }^{1}$ In order to show the coincidence between our results and those in the first-quantization, we recast the amplitudes into a BRST invariant form in terms of the conformal gauge worldsheet theory. For this purpose, together with the superreparametrization ghosts, we introduce the longitudinal variables with nonstandard interactions, which is the supersymmetric $X^{ \pm}$ CFT constructed in [9] with the identification $\frac{d-10}{8}=-Q^{2}$.

The organization of this paper is as follows. In section 2, we recapitulate the lightcone gauge superstring field theory in the linear dilaton background constructed in [20]. In section 3, we show that the multiloop amplitudes can be expressed in terms of a BRST invariant worldsheet theory in the conformal gauge. For this purpose, we add the supersymmetric $X^{ \pm}$CFT and superreparametrization ghosts to the worldsheet theory. We show that the supercurrents in the light-cone gauge, inserted at the interaction points, can be transformed into the picture changing operators (PCO's) in the conformal gauge and the expressions become BRST invariant. In section 4, we carry out the analytic continuation $Q \rightarrow 0$ of the Feynman amplitudes. We show that the results from the first-quantized formalism are reproduced. Namely, the results obtained here coincide with those obtained using the Sen-Witten prescription [24-26], up to infrared divergence problems. Section 5 is devoted to conclusions and discussions. In appendix A, the definitions of the Arakelov metric and Arakelov Green's functions are presented. In appendix B, some details of the supersymmetric $X^{ \pm}$CFT are given. Formulas used in the text are provided in appendices $\mathrm{C}$ and $\mathrm{D}$.

\section{Superstring field theory in linear dilaton background}

In this section, we review the light-cone gauge superstring field theory in linear dilaton background constructed in [20]. The string field theory is given for Type II superstring theory formulated in the NSR formalism. The heterotic case can be dealt with in a similar way.

\footnotetext{
${ }^{1}$ In [21-23], Sen has constructed covariant string field theories which reproduce the Feynman amplitudes from the first-quantized approach.
} 


\subsection{Linear dilaton background}

In order to regularize various divergences, we consider the superstring theory in a linear dilaton background $\Phi=-i Q X^{1}$, with a real constant $Q$. The worldsheet action of $X^{1}$ and its fermionic partners $\psi^{1}, \bar{\psi}^{1}$ on a worldsheet with metric $d s^{2}=2 \hat{g}_{z \bar{z}} d z d \bar{z}$ becomes

$$
\begin{gathered}
S\left[X^{1}, \psi^{1}, \bar{\psi}^{1} ; \hat{g}_{z \bar{z}}\right]=\frac{1}{8 \pi} \int d z \wedge d \bar{z} \sqrt{\hat{g}}\left(\hat{g}^{a b} \partial_{a} X^{1} \partial_{b} X^{1}-2 i Q \hat{R} X^{1}\right) \\
+\frac{1}{4 \pi} \int d z \wedge d \bar{z} i\left(\psi^{1} \bar{\partial} \psi^{1}+\bar{\psi}^{1} \partial \bar{\psi}^{1}\right)
\end{gathered}
$$

and the energy-momentum tensor and the supercurrent, which generate the superconformal transformations, are given as

$$
\begin{aligned}
T^{X^{1}}(z)= & -\frac{1}{2}\left(\partial X^{1}\right)^{2}-i Q\left(\partial-\partial \ln \hat{g}_{z \bar{z}}\right) \partial X^{1}-\frac{1}{2} \psi^{1} \partial \psi^{1} \\
& -Q^{2}\left(-\frac{1}{2}\left(\partial \ln g_{z \bar{z}}\right)^{2}+\partial^{2} \ln g_{z \bar{z}}\right) \\
T_{F}^{X^{1}}(z)= & -\frac{i}{2} \partial X^{1} \psi^{1}+Q\left(\partial-\frac{1}{2} \partial \ln \hat{g}_{z \bar{z}}\right) \psi^{1} .
\end{aligned}
$$

In order to construct string field theory and calculate amplitudes we need the correlation functions of the linear dilaton conformal field theory. Since the fermionic part is just a free theory, we concentrate on the bosonic part. Defining

$$
\tilde{X}^{1} \equiv X^{1}-i Q \ln \left(2 g_{z \bar{z}}\right),
$$

the correlation function of $e^{i p_{r} \tilde{X}^{1}}\left(Z_{r}, \bar{Z}_{r}\right)(r=1, \cdots, N)$ can be calculated on a Riemann surface [20]. Using the Arakelov metric $d s^{2}=2 g_{z \bar{z}}^{\mathrm{A}} d z d \bar{z}$ [27] defined on the surface, it is given as

$$
\begin{aligned}
\int & {\left[d X^{1}\right]_{g_{z \bar{z}}} e^{-S\left[X^{1} ; g_{z \bar{z}}\right]} \prod_{r=1}^{N} e^{i p_{r} \tilde{X}^{1}}\left(Z_{r}, \bar{Z}_{r}\right) } \\
= & 2 \pi \delta\left(\sum_{r} p_{r}+2 Q(1-g)\right) \\
& \times e^{-\frac{1-12 Q^{2}}{24} \Gamma\left[\sigma ; g_{z \bar{z}}^{\mathrm{A}}\right]} Z^{X}\left[g_{z \bar{z}}^{\mathrm{A}}\right] \prod_{r>s} e^{-p_{r} p_{s} G^{\mathrm{A}}\left(Z_{r}, Z_{s}\right)} \prod_{r}\left(2 g_{z \bar{z}}^{\mathrm{A}}\right)^{\frac{1}{2} p_{r}^{2}+Q p_{r}},
\end{aligned}
$$

where $Z^{X}\left[g_{z \bar{z}}^{\mathrm{A}}\right]$ denotes the partition function for a free boson on the worldsheet with the Arakelov metric (C.22), and

$$
\begin{aligned}
& S\left[X^{1} ; g_{a b}\right]=\frac{1}{8 \pi} \int d z \wedge d \bar{z} \sqrt{g}\left(g^{a b} \partial_{a} X^{1} \partial_{b} X^{1}-2 i Q R X^{1}\right) \\
& \sigma=\ln g_{z \bar{z}}-\ln g_{z \bar{z}}^{\mathrm{A}} \\
& \Gamma\left[\sigma ; g_{a b}\right]=-\frac{1}{4 \pi} \int d z \wedge d \bar{z} \sqrt{g}\left(g^{a b} \partial_{a} \sigma \partial_{b} \sigma+2 R \sigma\right) .
\end{aligned}
$$


$G^{\mathrm{A}}(z, w)$ denotes the Arakelov Green's function for the Arakelov metric. The definitions of $g_{z \bar{z}}^{\mathrm{A}}$ and $G^{\mathrm{A}}(z, w)$ are summarized in appendix A. The anomaly factor $e^{-\frac{1-12 Q^{2}}{24} \Gamma\left[\sigma ; \hat{g}_{z \bar{z}}\right]}$ is exactly what we expect for a theory with the central charge

$$
c=1-12 Q^{2}
$$

of the linear dilaton conformal field theory.

$e^{i p \tilde{X}^{1}}$ thus defined turns out to be a primary field with conformal dimension

$$
\frac{1}{2} p^{2}+Q p=\frac{1}{2}(p+Q)^{2}-\frac{Q^{2}}{2} .
$$

Notice that $\tilde{X}^{1}$ satisfies

$$
\partial \bar{\partial} \tilde{X}^{1}=0
$$

if there are no source terms, and thus $i \partial \tilde{X}^{1}(z), i \bar{\partial} \tilde{X}^{1}(\bar{z})$ can be expanded as

$$
\begin{aligned}
& i \partial \tilde{X}^{1}(z)=\sum_{n} \alpha_{n}^{1} z^{-n-1}, \\
& i \bar{\partial} \tilde{X}^{1}(\bar{z})=\sum_{n} \bar{\alpha}_{n}^{1} \bar{z}^{-n-1},
\end{aligned}
$$

where $\alpha_{n}^{1}$ and $\bar{\alpha}_{n}^{1}$ satisfy the canonical commutation relations. The states in the CFT are given as linear combinations of the Fock space states

$$
\alpha_{-n_{1}}^{1} \cdots \alpha_{-n_{k}}^{1} \bar{\alpha}_{-\bar{n}_{1}}^{1} \cdots \bar{\alpha}_{-\bar{n}_{l}}^{1}|p\rangle
$$

where $|p\rangle=e^{i p \tilde{X}^{1}}(0)|0\rangle$. The states and the oscillators satisfy

$$
\begin{aligned}
\left\langle p_{1} \mid p_{2}\right\rangle & =2 \pi \delta\left(p_{1}+p_{2}+2 Q\right), \\
\left(\alpha_{n}^{1}\right)^{*} & =-\left(\alpha_{-n}^{1}+2 Q \delta_{n, 0}\right) \\
\left(\bar{\alpha}_{n}^{1}\right)^{*} & =-\left(\bar{\alpha}_{-n}^{1}+2 Q \delta_{n, 0}\right)
\end{aligned}
$$

where $\langle p|,\left(\alpha_{n}^{1}\right)^{*},\left(\bar{\alpha}_{n}^{1}\right)^{*}$ are the BPZ conjugates of $|p\rangle, \alpha_{n}^{1}, \bar{\alpha}_{n}^{1}$ respectively. On the sphere, the correlation function is given by using the worldsheet metric $d s^{2}=d z d \bar{z}$ on the complex plane as

$$
\begin{aligned}
& \int\left[d X^{1}\right]_{g_{z \bar{z}}} e^{-S\left[X^{1} ; g_{z \bar{z}}\right]} \prod_{r=1}^{N} e^{i p_{r} \tilde{X}^{1}}\left(Z_{r}, \bar{Z}_{r}\right) \\
& =2 \pi \delta\left(\sum_{r} p_{r}+2 Q\right) e^{-\frac{1-12 Q^{2}}{24} \Gamma\left[\sigma ; \frac{1}{2}\right]} \prod_{r>s}\left|Z_{r}-Z_{s}\right|^{2 p_{r} p_{s}} .
\end{aligned}
$$

Using these, it is straightforward to construct the light-cone gauge superstring field theory action in the background. 


\subsection{Light-cone gauge superstring field theory in linear dilaton background}

Let us construct the light-cone gauge superstring field theory based on the worldsheet theory with the variables

$$
X^{i}, \psi^{i}, \bar{\psi}^{i} \quad(i=1, \cdots, 8),
$$

where the action for $X^{1}, \psi^{1}, \bar{\psi}^{1}$ is taken to be (2.1) and that for other variables is the free one. The worldsheet theory of the transverse variables turns out to be a superconformal field theory with central charge

$$
c=12-12 Q^{2}
$$

The string field

$$
|\Phi(t, \alpha)\rangle
$$

is taken to be an element of the Hilbert space of the transverse variables on the worldsheet and a function of

$$
\begin{aligned}
t & =x^{+}, \\
\alpha & =2 p^{+}
\end{aligned}
$$

$|\Phi(t, \alpha)\rangle$ should be GSO even and satisfy the level-matching condition

$$
\left(L_{0}-\bar{L}_{0}\right)|\Phi(t, \alpha)\rangle=0,
$$

where $L_{0}, \bar{L}_{0}$ are the zero modes of the Virasoro generators of the worldsheet theory.

The action of the string field theory is given by $[7,11]$

$$
\begin{aligned}
S & =\int d t\left[\frac{1}{2} \sum_{\mathrm{B}} \int_{-\infty}^{\infty} \frac{\alpha d \alpha}{4 \pi}\left\langle\Phi_{\mathrm{B}}(-\alpha)\left|\left(i \partial_{t}-\frac{L_{0}+\bar{L}_{0}-1+Q^{2}-i \varepsilon}{\alpha}\right)\right| \Phi_{\mathrm{B}}(\alpha)\right\rangle\right. \\
& +\frac{1}{2} \sum_{\mathrm{F}} \int_{-\infty}^{\infty} \frac{d \alpha}{4 \pi}\left\langle\Phi_{\mathrm{F}}(-\alpha)\left|\left(i \partial_{t}-\frac{L_{0}+\bar{L}_{0}-1+Q^{2}-i \varepsilon}{\alpha}\right)\right| \Phi_{\mathrm{F}}(\alpha)\right\rangle \\
& -\frac{g_{s}}{6} \sum_{\mathrm{B}_{1}, \mathrm{~B}_{2}, \mathrm{~B}_{3}} \int \prod_{r=1}^{3}\left(\frac{\alpha_{r} d \alpha_{r}}{4 \pi}\right) \delta\left(\sum_{r=1}^{3} \alpha_{r}\right)\left\langle V_{3} \mid \Phi_{\mathrm{B}_{1}}\left(\alpha_{1}\right)\right\rangle\left|\Phi_{\mathrm{B}_{2}}\left(\alpha_{2}\right)\right\rangle\left|\Phi_{\mathrm{B}_{3}}\left(\alpha_{3}\right)\right\rangle \\
& \left.-\frac{g_{s}}{2} \sum_{\mathrm{B}_{1}, \mathrm{~F}_{2}, \mathrm{~F}_{3}} \int \prod_{r=1}^{3}\left(\frac{\alpha_{r} d \alpha_{r}}{4 \pi}\right) \delta\left(\sum_{r=1}^{3} \alpha_{r}\right)\left\langle V_{3} \mid \Phi_{\mathrm{B}_{1}}\left(\alpha_{1}\right)\right\rangle \alpha_{2}^{-\frac{1}{2}}\left|\Phi_{\mathrm{F}_{2}}\left(\alpha_{2}\right)\right\rangle \alpha_{3}^{-\frac{1}{2}}\left|\Phi_{\mathrm{F}_{3}}\left(\alpha_{3}\right)\right\rangle\right] .
\end{aligned}
$$

The first and the second terms are the kinetic terms with the Feynman $i \varepsilon$ and $\langle\Phi(-\alpha)|$ denotes the BPZ conjugate of $|\Phi(-\alpha)\rangle$. The third and the fourth terms are the three string vertices and $g_{s}$ is the string coupling constant. $\sum_{\mathrm{B}}$ and $\sum_{\mathrm{F}}$ denote the sums over bosonic and fermionic string fields respectively. By the state-operator correspondence of the worldsheet conformal field theory, there exists a local operator $\mathcal{O}_{\Phi}(w, \bar{w})$ corresponding to any state $|\Phi\rangle .\left\langle V_{3} \mid \Phi\left(\alpha_{1}\right)\right\rangle\left|\Phi\left(\alpha_{2}\right)\right\rangle\left|\Phi\left(\alpha_{3}\right)\right\rangle$ with $\sum_{r=1}^{3} \alpha_{r}=0$ is defined to be

$$
\begin{aligned}
& \left\langle V_{3} \mid \Phi\left(\alpha_{1}\right)\right\rangle\left|\Phi\left(\alpha_{2}\right)\right\rangle\left|\Phi\left(\alpha_{3}\right)\right\rangle \\
& =\left\langle\lim _{\rho \rightarrow \rho_{0}}\left|\rho-\rho_{0}\right|^{\frac{3}{2}} T_{F}^{L C}(\rho) \bar{T}_{F}^{L C}(\bar{\rho}) h_{1} \circ \mathcal{O}_{\Phi\left(\alpha_{1}\right)}(0,0) h_{2} \circ \mathcal{O}_{\Phi\left(\alpha_{2}\right)}(0,0) h_{3} \circ \mathcal{O}_{\Phi\left(\alpha_{3}\right)}(0,0)\right\rangle_{\Sigma},
\end{aligned}
$$




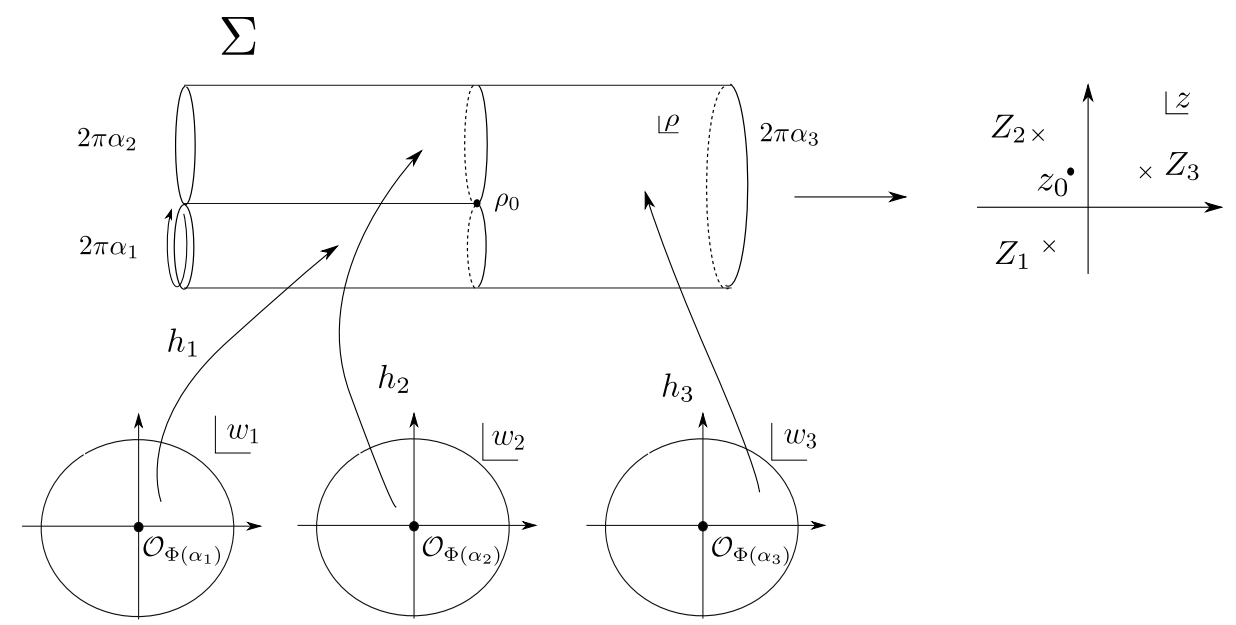

Figure 1. The three string vertex for superstrings. Here we consider the case $\alpha_{1}, \alpha_{2}>0, \alpha_{3}<0$.

in terms of a correlation function on $\Sigma$, which is the worldsheet describing the three string interaction depicted in figure 1. On each cylinder corresponding to an external line, one can introduce a complex coordinate

$$
\rho=\tau+i \sigma,
$$

whose real part $\tau$ coincides with the Wick rotated light-cone time it and imaginary part $\sigma \sim \sigma+2 \pi \alpha_{r}$ parametrizes the closed string at each time. The $\rho$ 's on the cylinders are smoothly connected except at the interaction point $\rho_{0}$ and we get a complex coordinate $\rho$ on $\Sigma$. The correlation function \langle\rangle$_{\Sigma}$ is defined with the metric

$$
d s^{2}=d \rho d \bar{\rho},
$$

on the worldsheet. $h_{r}\left(w_{r}\right)$ gives a map from a unit disk $\left|w_{r}\right|<1$ to the cylinder corresponding to the $r$-th external line so that

$$
w_{r}=e^{\frac{1}{\alpha_{r}}\left(h_{r}\left(w_{r}\right)-\rho_{0}\right)} .
$$

$T_{F}^{L C}, \bar{T}_{F}^{L C}$ are the supercurrents of the transverse worldsheet theory.

\subsection{Feynman amplitudes of light-cone gauge superstring field theory in linear dilaton background}

It is straightforward to calculate the amplitudes by the old-fashioned perturbation theory starting from the action (2.16) and Wick rotate to Euclidean time. The propagator and the vertex are given by the worldsheets depicted in figure 2. Each term in the expansion corresponds to a light-cone gauge Feynman diagram for strings. A typical diagram is depicted in figure 3 .

A Wick rotated $g$-loop $N$-string diagram is conformally equivalent to an $N$ punctured genus $g$ Riemann surface $\Sigma$. A $g$-loop $N$-string amplitude is given as an integral over the moduli space of $\Sigma$ as $[28,29]$

$$
\mathcal{A}_{N}^{(g)}=\left(i g_{s}\right)^{2 g-2+N} C \int[d T][\alpha d \theta][d \alpha] F_{N}^{(g)},
$$




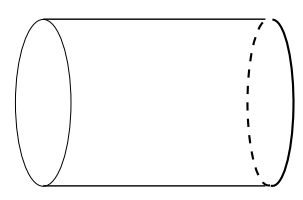

propagator

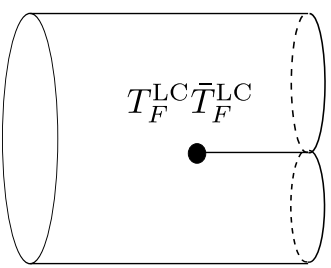

vertex

Figure 2. The propagator and the vertex of the string field theory.

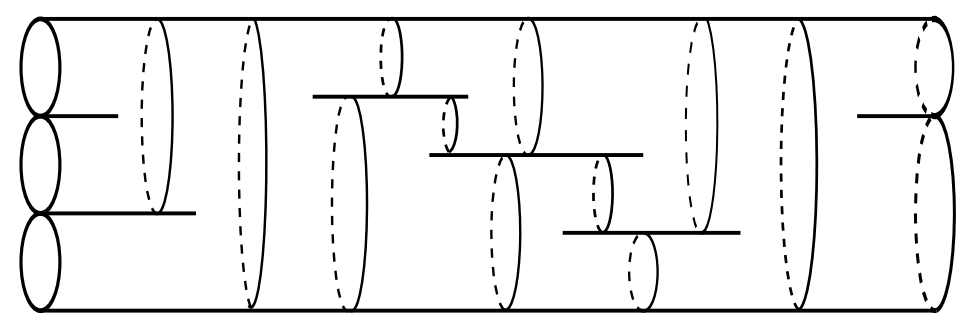

Figure 3. A string diagram with 3 incoming, 2 outgoing strings and 3 loops.

where $\int[d T][\alpha d \theta][d \alpha]$ denotes the integration over the moduli parameters and $C$ is the combinatorial factor. In each channel, the integration measure is given as

$$
\int[d T][\alpha d \theta][d \alpha]=\prod_{a=1}^{2 g-3+N}\left(-i \int_{0}^{\infty} d T_{a}\right) \prod_{A=1}^{g} \int \frac{d \alpha_{A}}{4 \pi} \prod_{\mathcal{I}=1}^{3 g-3+N}\left(\left|\alpha_{\mathcal{I}}\right| \int_{0}^{2 \pi} \frac{d \theta_{\mathcal{I}}}{2 \pi}\right) .
$$

Here $T_{a}$ 's are heights of the cylinders corresponding to internal lines, $\alpha_{A}$ 's denote the circumferences of the cylinders corresponding to the + components of the loop momenta and $\alpha_{\mathcal{I}}$ 's and $\theta_{\mathcal{I}}$ 's are the string-lengths and the twist angles for the internal propagators.

The integrand $F_{N}^{(g)}$ is given as a path integral over the transverse variables $X^{i}, \psi^{i}, \bar{\psi}^{i}$ $(i=1, \ldots, 8)$ on the light-cone diagram. A light-cone diagram consists of cylinders which correspond to propagators of the closed string as mentioned above. On each cylinder, one can introduce a complex coordinate $\rho$ in the same way as the complex coordinate (2.18) is introduced on the three string vertex. The $\rho$ 's on the cylinders are smoothly connected except at the interaction points and we get a complex coordinate $\rho$ on the light-cone diagram $\Sigma$. The path integral on the light-cone diagram is defined by using the metric (2.19).

$\rho$ is not a good coordinate around the interaction points and the punctures, and the metric (2.19) is not well-defined at these points. $F_{N}^{(g)}$ can be expressed in terms of correlation functions defined with a metric $d \hat{s}^{2}=2 \hat{g}_{z \bar{z}} d z d \bar{z}$ which is regular everywhere on the worldsheet, as

$$
\begin{aligned}
& F_{N}^{(g)}=(2 \pi)^{2} \delta\left(\sum_{r=1}^{N} p_{r}^{+}\right) \delta\left(\sum_{r=1}^{N} p_{r}^{-}\right) e^{-\frac{1}{2}\left(1-Q^{2}\right) \Gamma\left[\sigma ; \hat{g}_{z \bar{z}}\right]} \\
& \quad \times \int\left[d X^{i} d \psi^{i} d \bar{\psi}^{i}\right]_{\hat{g}_{z \bar{z}}} e^{-S^{\mathrm{LC}}\left[X^{i}, \psi^{i}, \bar{\psi}^{i}\right]} \prod_{I=1}^{2 g-2+N}\left(\left|\partial^{2} \rho\left(z_{I}\right)\right|^{-\frac{3}{2}} T_{F}^{\mathrm{LC}}\left(z_{I}\right) \bar{T}_{F}^{\mathrm{LC}}\left(\bar{z}_{I}\right)\right) \prod_{r=1}^{N} V_{r}^{\mathrm{LC}} .
\end{aligned}
$$


Here $S^{\mathrm{LC}}\left[X^{i}, \psi^{i}, \bar{\psi}^{i}\right]$ denotes the worldsheet action of the transverse variables and the path integral measure $\left[d X^{i} d \psi^{i} d \bar{\psi}^{i}\right]_{\hat{g}_{z \bar{z}}}$ is defined with the metric $d \hat{s}^{2}=2 \hat{g}_{z \bar{z}} d z d \bar{z}$. Since the integrand should be defined by using the canonical metric $d s^{2}=\partial \rho \bar{\partial} \bar{\rho} d z d \bar{z}$ on the light-cone diagram, we need the anomaly factor $e^{-\frac{1}{2}\left(1-Q^{2}\right) \Gamma\left[\sigma ; \hat{g}_{z \bar{z}}\right]}$, where

$$
\begin{aligned}
& \sigma=\ln \partial \rho \bar{\partial} \bar{\rho}-\ln \hat{g}_{z \bar{z}} \\
& \Gamma\left[\sigma ; \hat{g}_{z \bar{z}}\right]=-\frac{1}{4 \pi} \int d z \wedge d \bar{z} \sqrt{\hat{g}}\left(\hat{g}^{a b} \partial_{a} \sigma \partial_{b} \sigma+2 \hat{R} \sigma\right) .
\end{aligned}
$$

$V_{r}^{\mathrm{LC}}$ denotes the vertex operator for the $r$-th external line. When the $r$-th external line corresponds to the state

$$
\alpha_{-n_{1}}^{i_{1}} \cdots \bar{\alpha}_{-\bar{n}_{1}}^{\bar{i}_{1}} \cdots \psi_{-s_{1}}^{j_{1}} \cdots \bar{\psi}_{-\bar{s}_{1}}^{\bar{j}_{1}} \cdots\left|p_{r}\right\rangle
$$

in the (NS,NS) sector, the light-cone vertex $V_{r}^{\mathrm{LC}}$ is given as

$$
\begin{aligned}
V_{r}^{\mathrm{LC}}=\alpha_{r} & \oint_{0} \frac{d w_{r}}{2 \pi i} i \partial \tilde{X}^{i_{1}}\left(w_{r}\right) w_{r}^{-n_{1}} \cdots \oint_{0} \frac{d \bar{w}_{r}}{2 \pi i} i \bar{\partial} \tilde{X}^{\bar{i}_{1}}\left(\bar{w}_{r}\right) \bar{w}_{r}^{-\bar{n}_{1}} \ldots \\
& \times \oint_{0} \frac{d w_{r}}{2 \pi i} \psi^{j_{1}}\left(w_{r}\right) w_{r}^{-s_{1}-\frac{1}{2}} \cdots \oint_{0} \frac{d \bar{w}_{r}}{2 \pi i} \psi^{\bar{j}_{1}}\left(\bar{w}_{r}\right) \bar{w}_{r}^{-\bar{s}_{1}-\frac{1}{2}} \cdots \\
& \times e^{i \vec{p}_{r} \cdot \overrightarrow{\tilde{X}}}\left(w_{r}=0, \bar{w}_{r}=0\right) e^{-p_{r}^{-} \tau_{0}^{(r)}} .
\end{aligned}
$$

Here

$$
\begin{aligned}
\tilde{X}^{i} & \equiv X^{i}-i Q \delta^{i 1} \ln \left(2 g_{z \bar{z}}\right) \\
w_{r} & \equiv \exp \left[\frac{1}{\alpha_{r}}\left(\rho(z)-\rho\left(z_{I^{(r)}}\right)\right)\right], \\
\tau_{0}^{(r)} & \equiv \operatorname{Re} \rho\left(z_{I^{(r)}}\right)
\end{aligned}
$$

and $z_{I^{(r)}}$ is defined to be the coordinate of the interaction point at which the $r$-th external line interacts. The on-shell and the level-matching conditions are

$$
\frac{1}{2}\left(-2 p_{r}^{+} p_{r}^{-}+p_{r}^{i} p_{r}^{i}\right)+Q p_{r}^{1}+\mathcal{N}_{r}=\frac{1}{2}\left(1-Q^{2}\right), \quad \mathcal{N}_{r} \equiv \sum_{k} n_{k}+\sum_{l} s_{l}=\sum_{\bar{k}} \bar{n}_{\bar{k}}+\sum_{\bar{l}} \bar{s}_{\bar{l}} .
$$

It is possible to calculate the right hand side of (2.23). $\rho$ can be given as a function of local coordinate $z$ on $\Sigma$ as

$$
\rho(z)=\sum_{r=1}^{N} \alpha_{r}\left[\ln E\left(z, Z_{r}\right)-2 \pi i \int_{P_{0}}^{z} \omega \frac{1}{\operatorname{Im} \Omega} \operatorname{Im} \int_{P_{0}}^{Z_{r}} \omega\right], \quad \sum_{r=1}^{N} \alpha_{r}=0,
$$

up to an additive constant independent of $z$. Here $E(z, w)$ is the prime form of the surface, $\omega$ is the canonical basis of the holomorphic abelian differentials and $\Omega$ is the period matrix. ${ }^{2}$ The base point $P_{0}$ is arbitrary. There are $2 g-2+N$ zeros of $\partial \rho$ and we denote them by $z_{I}(I=1, \cdots, 2 g-2+N)$. They correspond to the interaction points of the light-cone

\footnotetext{
${ }^{2}$ For the mathematical background relevant for string perturbation theory, we refer the reader to [30].
} 
diagram. Substituting (2.30) into (2.24) yields a divergent result for $\Gamma\left[\sigma ; \hat{g}_{z \bar{z}}\right]$. We can obtain $e^{-\Gamma\left[\sigma ; \hat{g}_{z \bar{z}}\right]}$ up to a divergent numerical factor by regularizing it as was done in [31]. The divergent factor can be absorbed in a redefinition of $g_{s}$ and the vertex operator. Taking $\hat{g}_{z \bar{z}}$ to be the Arakelov metric [27], $e^{-\Gamma\left[\sigma ; g_{z \bar{z}}^{\mathrm{A}}\right]}$ for higher genus surfaces is calculated in [13] to be

$$
e^{-\Gamma\left[\sigma ; g_{z \bar{z}}^{\mathrm{A}}\right]} \propto e^{-W} \prod_{r} e^{-2 \operatorname{Re} \bar{N}_{00}^{r r}} \prod_{I}\left|\partial^{2} \rho\left(z_{I}\right)\right|^{-3},
$$

up to a numerical constant which can be fixed by imposing the factorization condition. Here

$$
\begin{aligned}
-W \equiv & -2 \sum_{I<J} G^{\mathrm{A}}\left(z_{I} ; z_{J}\right)-2 \sum_{r<s} G^{\mathrm{A}}\left(Z_{r} ; Z_{s}\right)+2 \sum_{I, r} G^{\mathrm{A}}\left(z_{I} ; Z_{r}\right) \\
& -\sum_{r} \ln \left(2 g_{Z_{r} \bar{Z}_{r}}^{\mathrm{A}}\right)+3 \sum_{I} \ln \left(2 g_{z_{I} \bar{z}_{I}}^{\mathrm{A}}\right) \\
\bar{N}_{00}^{r r} \equiv & \lim _{z \rightarrow Z_{r}}\left[\frac{\rho\left(z_{I^{(r)}}\right)-\rho(z)}{\alpha_{r}}+\ln \left(z-Z_{r}\right)\right] \\
= & \frac{\rho\left(z_{I^{(r)}}\right)}{\alpha_{r}}-\sum_{s \neq r} \frac{\alpha_{s}}{\alpha_{r}} \ln E\left(Z_{r}, Z_{s}\right)+\frac{2 \pi i}{\alpha_{r}} \int_{P_{0}}^{Z_{r}} \omega \frac{1}{\operatorname{Im} \Omega} \sum_{s=1}^{N} \alpha_{s} \operatorname{Im} \int_{P_{0}}^{Z_{s}} \omega .
\end{aligned}
$$

The correlation functions of $X^{i}, \psi^{i}, \bar{\psi}^{i}$ which appear in (2.23) can be calculated by using the formulas given in [32-36] and (2.4). From the explicit form of the integrand $F_{N}^{(g)}$, one can see that the amplitude $A_{N}^{(g)}$ suffers from divergences due to the collisions of the interaction points and the degenerations of the surface $\Sigma$, if $Q=0$. In [20], it was shown that $A_{N}^{(g)}$ becomes finite if one takes $Q^{2}>10$ and $\varepsilon>0$. Therefore it is possible to define $A_{N}^{(g)}$ as an analytic function of $Q$ and take the limit $Q \rightarrow 0$ as is usually done in dimensional regularization of field theory.

\section{BRST invariant form of the amplitudes}

We would like to show that the integrand (2.23) can be rewritten in terms of a correlation function of the conformal gauge variables $X^{\mu}, \psi^{\mu}, \bar{\psi}^{\mu}(\mu=+,-, 1, \ldots, 8), b, c, \bar{b}, \bar{c}, \beta, \gamma, \bar{\beta}, \bar{\gamma}$. For $Q \neq 0$, the worldsheet theory of the longitudinal variables $X^{ \pm}, \psi^{ \pm}, \bar{\psi}^{ \pm}$becomes socalled $X^{ \pm}$CFT, which is defined and analyzed in [9, 14]. The results of these references are summarized in appendix B. Using (B.15) and (B.16) there, one can prove

$$
\begin{aligned}
(2 \pi)^{2} \delta & \left(\sum_{r=1}^{N} p_{r}^{+}\right) \delta\left(\sum_{r=1}^{N} p_{r}^{-}\right)\left(Z^{X}\left[\hat{g}_{z \bar{z}}\right] Z^{\psi}\left[\hat{g}_{z \bar{z}}\right]\right)^{2} \prod_{r=1}^{N} V_{r}^{\mathrm{LC}} \\
=\prod_{r=1}^{N}( & \left.\alpha_{r} e^{\operatorname{Re} \bar{N}_{00}^{r r}}\right) e^{-\frac{Q^{2}}{2} \Gamma\left[\sigma ; \hat{g}_{z \bar{z}}\right]} \int\left[d \mathcal{X}^{+} d \mathcal{X}^{-}\right]_{\hat{g}_{z \bar{z}}} e^{-S_{\text {super }}^{ \pm}\left[\mathcal{X}^{ \pm} ; \hat{g}_{z \bar{z}}\right]} \\
& \times \prod_{r=1}^{N}\left[\oint_{z_{I}(r)} \frac{d \mathbf{z}}{2 \pi i} \mathcal{S}\left(\mathbf{z}, Z_{r}\right) \oint_{\bar{z}_{I}(r)} \frac{d \overline{\mathbf{z}}}{2 \pi i} \overline{\mathcal{S}}\left(\overline{\mathbf{z}}, \bar{Z}_{r}\right) V_{r}^{\mathrm{DDF}}\left(Z_{r}, \bar{Z}_{r}\right)\right] .
\end{aligned}
$$

Here $\mathcal{X}^{ \pm}$is the superfield given in (B.3). The supersymmetric contour integral is defined as

$$
\oint_{z_{I^{(r)}}} \frac{d \mathbf{z}}{2 \pi i}=\oint_{z_{I^{(r)}}} \frac{d z}{2 \pi i} \int d \theta
$$


using the Berezinian integral $\int d \theta$, and the antiholomorphic version is defined similarly. $\mathcal{S}(\mathbf{z}, w)$ is defined to be

$$
\mathcal{S}(\mathbf{z}, w) \equiv D \ln \left(\partial \mathcal{X}^{+}-\frac{\partial D \mathcal{X}^{+} D \mathcal{X}^{+}}{\partial \mathcal{X}^{+}}\right)(\mathbf{z}) e^{-i \frac{Q^{2}}{\alpha_{r}}\left(\mathcal{X}_{L}^{+}(\mathbf{z})-X_{L}^{+}(w)\right)},
$$

and $\overline{\mathcal{S}}(\overline{\mathbf{z}}, w)$ is the antiholomorphic version. $V_{r}^{\mathrm{DDF}}\left(Z_{r}, \bar{Z}_{r}\right)$ is the supersymmetric DDF vertex operator given by

$V_{r}^{\mathrm{DDF}}\left(Z_{r}, \bar{Z}_{r}\right)=A_{-n_{1}}^{i_{1}(r)} \cdots \bar{A}_{-\bar{n}_{1}}^{\bar{i}_{1}(r)} \cdots B_{-s_{1}}^{j_{1}(r)} \cdots \bar{B}_{-\bar{s}_{1}}^{\bar{j}_{1}(r)} \cdots e^{-i p_{r}^{+} X^{-}-i\left(p_{r}^{-}-\frac{\mathcal{N}_{r}}{p_{r}^{+}}\right) X^{+}+i p_{r}^{i} \tilde{X}^{i}}\left(Z_{r}, \bar{Z}_{r}\right)$,

with the DDF operators $A_{-n}^{i(r)}, B_{-s}^{j(r)}$ for the $r$-th string defined as

$$
\begin{aligned}
A_{-n}^{i(r)} & =\oint_{Z_{r}} \frac{d \mathbf{z}}{2 \pi i} i D\left(\tilde{\mathcal{X}}^{i}+i Q \delta^{i, 1} \Phi\right) e^{-i \frac{n}{p_{r}^{+}} \mathcal{X}_{L}^{+}}(\mathbf{z}), \\
B_{-s}^{i(r)} & =\oint_{Z_{r}} \frac{d \mathbf{z}}{2 \pi i} \frac{1}{\left(i p_{r}^{+}\right)^{\frac{1}{2}}} \Theta^{+} D\left(\tilde{\mathcal{X}}^{i}+i Q \delta^{i, 1} \Phi\right) e^{-i \frac{s}{p_{r}^{+}} \mathcal{X}_{L}^{+}}(\mathbf{z}),
\end{aligned}
$$

where $\tilde{\mathcal{X}}^{i}$ is the superfield for $\tilde{X}^{i}, \psi^{i}, \bar{\psi}^{i}, \Phi$ is defined in (B.5) and $\mathcal{X}_{L}^{+}$denotes the left moving part of $\mathcal{X}^{+} . \bar{A}_{-n}^{i(r)}, \bar{B}_{-s}^{i(r)}$ are similarly given for the antiholomorphic sector. The product $\mathcal{S} \overline{\mathcal{S}} V_{r}^{\mathrm{DDF}}$ is normal ordered as

$$
\begin{aligned}
& \mathcal{S}\left(\mathbf{z}, Z_{r}\right) \overline{\mathcal{S}}\left(\overline{\mathbf{z}}, \bar{Z}_{r}\right) V_{r}^{\mathrm{DDF}}\left(Z_{r}, \bar{Z}_{r}\right) \\
& \quad \equiv \lim _{w \rightarrow Z_{r}} \lim _{\bar{w} \rightarrow \bar{Z}_{r}} \mathcal{S}(\mathbf{z}, w) \overline{\mathcal{S}}(\overline{\mathbf{z}}, \bar{w}) V_{r}^{\mathrm{DDF}}\left(Z_{r}, \bar{Z}_{r}\right)\left|w-Z_{r}\right|^{-Q^{2}} .
\end{aligned}
$$

Substituting (3.1) into (2.23), we find that the integrand $F_{N}^{(g)}$ can be expressed as

$$
\begin{aligned}
F_{N}^{(g)} \propto & \prod_{r=1}^{N}\left(\alpha_{r} e^{\operatorname{Re} \bar{N}_{00}^{r r}}\right) \prod_{I}\left|\partial^{2} \rho\left(z_{I}\right)\right|^{-\frac{3}{2}} e^{-\frac{1}{2} \Gamma\left[g_{z \bar{z}}^{\mathrm{A}}, \ln |\partial \rho|^{2}\right]}\left(Z^{X}\left[g_{z \bar{z}}^{\mathrm{A}}\right] Z^{\psi}\left[g_{z \bar{z}}^{\mathrm{A}}\right]\right)^{-2} \\
& \times \int\left[d \mathcal{X}^{\mu}\right]_{\hat{g}_{z \bar{z}}} e^{-S_{\mathrm{super}}^{ \pm}\left[\mathcal{X}^{ \pm} ; \hat{g}_{z \bar{z}}\right]-S^{\mathrm{LC}}\left[X^{i}, \psi^{i}, \bar{\psi}^{i}\right]} \prod_{I}\left[T_{F}^{\mathrm{LC}}\left(z_{I}\right) \bar{T}_{F}^{\mathrm{LC}}\left(\bar{z}_{I}\right)\right] \\
& \times \prod_{r=1}^{N}\left[\oint_{z_{I^{(r)}}} \frac{d \mathbf{z}}{2 \pi i} \mathcal{S}\left(\mathbf{z}, Z_{r}\right) \oint_{\bar{z}_{I^{(r)}}} \frac{d \overline{\mathbf{z}}}{2 \pi i} \overline{\mathcal{S}}\left(\overline{\mathbf{z}}, \bar{Z}_{r}\right) V_{r}^{\mathrm{DDF}}\left(Z_{r}, \bar{Z}_{r}\right)\right] .
\end{aligned}
$$

We will further rewrite (3.7) by introducing the ghost variables. The identities satisfied by the ghost correlation functions which should be used here are summarized in appendix C. Taking the metric $\hat{g}_{z \bar{z}}$ to be the Arakelov metric $g_{z \bar{z}}^{\mathrm{A}}$ and using (3.7), (C.28) and (C.35), 
the amplitude (2.21) can be rewritten as

$$
\begin{aligned}
A_{N}^{(g)} \propto & \left.\int d T\right][d \alpha][\alpha d \theta] \\
& \times \int\left[d X^{\mu} d \psi^{\mu} d \bar{\psi}^{\mu} d b d \bar{b} d c d \bar{c} d \beta d \bar{\beta} d \gamma d \bar{\gamma}\right]_{g_{z \bar{z}}^{\mathrm{A}}} e^{-S^{\mathrm{tot}}} \\
& \times \prod_{K=1}^{6 g-6+2 N}\left[\oint_{C_{K}} \frac{d z}{\partial \rho} b_{z z}+\varepsilon_{K} \oint_{\bar{C}_{K}} \frac{d \bar{z}}{\bar{\partial} \bar{\rho}} b_{\bar{z} \bar{z}}\right] \prod_{I}\left[e^{\phi} T_{F}^{\mathrm{LC}}\left(z_{I}\right) e^{\bar{\phi}} \bar{T}_{F}^{\mathrm{LC}}\left(\bar{z}_{I}\right)\right] \\
& \times \prod_{r=1}^{N}\left[\oint_{z_{I^{(r)}}} \frac{d \mathbf{z}}{2 \pi i} \mathcal{S}\left(\mathbf{z}, Z_{r}\right) \oint_{\bar{z}_{I}(r)} \frac{d \overline{\mathbf{z}}}{2 \pi i} \overline{\mathcal{S}}\left(\overline{\mathbf{z}}, \bar{Z}_{r}\right) c \bar{c} e^{-\phi-\bar{\phi}} V_{r}^{\mathrm{DDF}}\left(Z_{r}, \bar{Z}_{r}\right)\right] .
\end{aligned}
$$

Here $S^{\text {tot }}$ denotes the worldsheet action for the variables $X^{\mu}, \psi^{\mu}, \bar{\psi}^{\mu}$ and the ghosts. It is shown in [9] that the worldsheet theory becomes a conformal field theory with vanishing central charge, and we can define the nilpotent BRST operator $Q_{\mathrm{B}}$. The quantities which appear in this expression would be BRST invariant, if $e^{\phi} T_{F}^{\mathrm{LC}}\left(z_{I}\right)$ and $e^{\bar{\phi}} \bar{T}_{F}^{\mathrm{LC}}\left(\bar{z}_{I}\right)$ were the PCO's. Actually (3.8) can be turned into a BRST invariant form as

$$
\begin{aligned}
A_{N}^{(g)} \propto & \left.\int d T\right][d \alpha][\alpha d \theta] \\
& \times \int\left[d X^{\mu} d \psi^{\mu} d \bar{\psi}^{\mu} d b d \bar{b} d c d \bar{c} d \beta d \bar{\beta} d \gamma d \bar{\gamma}\right]_{g_{z \bar{z}}^{\mathrm{A}}} e^{-S^{\mathrm{tot}}} \\
& \times \prod_{K=1}^{6 g-6+2 N}\left[\oint_{C_{K}} \frac{d z}{\partial \rho} b_{z z}+\varepsilon_{K} \oint_{\bar{C}_{K}} \frac{d \bar{z}}{\bar{\partial} \bar{\rho}} b_{\bar{z} \bar{z}}\right] \prod_{I}\left[X\left(z_{I}\right) \bar{X}\left(\bar{z}_{I}\right)\right] \\
& \times \prod_{r=1}^{N}\left[\oint_{z_{I^{(r)}}} \frac{d \mathbf{z}}{2 \pi i} \mathcal{S}\left(\mathbf{z}, Z_{r}\right) \oint_{\bar{z}_{I}(r)} \frac{d \overline{\mathbf{z}}}{2 \pi i} \overline{\mathcal{S}}\left(\overline{\mathbf{z}}, \bar{Z}_{r}\right) c \bar{c} e^{-\phi-\bar{\phi}} V_{r}^{\mathrm{DDF}}\left(Z_{r}, \bar{Z}_{r}\right)\right],
\end{aligned}
$$

where

$$
X(z)=\left[c \partial \xi-e^{\phi} T_{F}+\frac{1}{4} \partial b \eta e^{2 \phi}+\frac{1}{4} b\left(2 \partial \eta e^{2 \phi}+\eta \partial e^{2 \phi}\right)\right](z)
$$

is the PCO and $\bar{X}(\bar{z})$ is its antiholomorphic counterpart. Here $T_{F}$ denotes the supercurrent for $\mathcal{X}^{\mu}(\mu=+,-, 1, \ldots, 8)$. Since the PCO's and the contour integrals of $\mathcal{S}\left(\mathbf{z}, Z_{r}\right), \overline{\mathcal{S}}\left(\overline{\mathbf{z}}, \bar{Z}_{r}\right)$ do not commute, we need to be a little careful about the definition of the right hand side of (3.9). To be precise, the right hand side of (3.9) should be defined as

$$
\begin{aligned}
& \int[d T][d \alpha][\alpha d \theta] \\
& \times \int\left[d X^{\mu} d \psi^{\mu} d b d \bar{b} d c d \bar{c} d \beta d \bar{\beta} d \gamma d \bar{\gamma}\right]_{g_{z \bar{z}}^{\mathrm{A}}} e^{-S^{\mathrm{tot}}} \\
& \quad \times \prod_{K=1}^{6 g-6+2 N}\left[\oint_{C_{K}} \frac{d z}{\partial \rho} b_{z z}+\varepsilon_{K} \oint_{\bar{C}_{K}} \frac{d \bar{z}}{\bar{\partial} \bar{\rho}} b_{\bar{z} \bar{z}}\right] \lim _{\epsilon \rightarrow 0} \prod_{I}\left[X\left(z_{I}+2 \epsilon\right) \bar{X}\left(\bar{z}_{I}+2 \epsilon\right)\right] \\
& \quad \times \prod_{r=1}^{N}\left[\oint_{C_{r,|\epsilon|}} \frac{d \mathbf{z}}{2 \pi i} \mathcal{S}\left(\mathbf{z}, Z_{r}\right) \oint_{\bar{C}_{r,|\epsilon|}} \frac{d \overline{\mathbf{z}}}{2 \pi i} \overline{\mathcal{S}}\left(\overline{\mathbf{z}}, \bar{Z}_{r}\right) c \bar{c} e^{-\phi-\bar{\phi}} V_{r}^{\mathrm{DDF}}\left(Z_{r}, \bar{Z}_{r}\right)\right] .
\end{aligned}
$$


The contour $C_{r,|\epsilon|}$ is a circle with radius $|\epsilon|$ around $z=z_{I^{(r)}}$. One can show

$$
\begin{aligned}
& {\left[Q_{\mathrm{B}}, \oint_{C_{r,|\epsilon|}} \frac{d \mathbf{z}}{2 \pi i} \mathcal{S}\left(\mathbf{z}, Z_{r}\right) \oint_{\bar{C}_{r,|\epsilon|}} \frac{d \overline{\mathbf{z}}}{2 \pi i} \overline{\mathcal{S}}\left(\overline{\mathbf{z}}, \bar{Z}_{r}\right) c \bar{c} e^{-\phi-\bar{\phi}} V_{r}^{\mathrm{DDF}}\left(Z_{r}, \bar{Z}_{r}\right)\right]} \\
& =\oint_{C_{r,|\epsilon|}} \frac{d \mathbf{z}}{2 \pi i} \partial D C(\mathbf{z}) e^{-i \frac{Q^{2}}{\alpha_{r}}\left(\mathcal{X}_{L}^{+}(\mathbf{z})-X_{L}^{+}\left(Z_{r}\right)\right)} \oint_{\bar{C}_{r,|\epsilon|}} \frac{d \overline{\mathbf{z}}}{2 \pi i} \overline{\mathcal{S}}\left(\overline{\mathbf{z}}, \bar{Z}_{r}\right) c \bar{c} e^{-\phi-\bar{\phi}} V_{r}^{\mathrm{DDF}}\left(Z_{r}, \bar{Z}_{r}\right) \\
& \quad+\oint_{C_{r,|\epsilon|}} \frac{d \mathbf{z}}{2 \pi i} \mathcal{S}\left(\mathbf{z}, Z_{r}\right) \oint_{\bar{C}_{r,|\epsilon|}} \frac{d \overline{\mathbf{z}}}{2 \pi i} \bar{\partial} \bar{D} C(\overline{\mathbf{z}}) e^{-i \frac{Q^{2}}{\alpha_{r}}\left(\mathcal{X}_{R}^{+}(\overline{\mathbf{z}})-X_{R}^{+}\left(\bar{Z}_{r}\right)\right)} c \bar{c} e^{-\phi-\bar{\phi}} V_{r}^{\mathrm{DDF}}\left(Z_{r}, \bar{Z}_{r}\right),
\end{aligned}
$$

and with no operators inside $C_{r,|\epsilon|}, \bar{C}_{r,|\epsilon|}$, this vanishes. All the other factors are BRST invariant in the usual way and thus one can prove that (3.9) is a BRST invariant expression. When there exists no $r(r=1, \ldots, N)$ such that $z_{I}=z_{I^{(r)}}$, we can simply replace the limit

$$
\lim _{\epsilon \rightarrow 0}\left[X\left(z_{I}+2 \epsilon\right) \bar{X}\left(\bar{z}_{I}+2 \epsilon\right)\right]
$$

by $X\left(z_{I}\right) \bar{X}\left(\bar{z}_{I}\right)$. If $z_{I}=z_{I^{(r)}}$ and there are no $r^{\prime} \neq r$ such that $z_{I}=z_{I^{\left(r^{\prime}\right)}}$, we obtain

$$
\begin{aligned}
\lim _{\epsilon \rightarrow 0} & {\left[X\left(z_{I}+2 \epsilon\right) \bar{X}\left(\bar{z}_{I}+2 \epsilon\right)\right] \oint_{C_{r, \mid \epsilon \epsilon}} \frac{d \mathbf{z}}{2 \pi i} \mathcal{S}\left(\mathbf{z}, Z_{r}\right) \oint_{\bar{C}_{r,|\epsilon|}} \frac{d \overline{\mathbf{z}}}{2 \pi i} \overline{\mathcal{S}}\left(\overline{\mathbf{z}}, \bar{Z}_{r}\right) c \bar{c} e^{-\phi-\bar{\phi}} V_{r}^{\mathrm{DDF}}\left(Z_{r}, \bar{Z}_{r}\right) } \\
= & {\left[\oint_{z_{I}} \frac{d \mathbf{z}}{2 \pi i} \mathcal{S}\left(\mathbf{z}, Z_{r}\right) X\left(z_{I}\right)-\frac{Q^{2}}{2} \frac{1}{p_{r}^{+}} e^{\phi} \partial\left(\psi^{+} e^{-i \frac{Q^{2}}{\alpha_{r}} X_{L}^{+}}\right)\left(z_{I}\right)\right] } \\
& \times\left[\oint_{\bar{z}_{I}} \frac{d \overline{\mathbf{z}}}{2 \pi i} \overline{\mathcal{S}}\left(\overline{\mathbf{z}}, \bar{Z}_{r}\right) \bar{X}\left(\bar{z}_{I}\right)-\frac{Q^{2}}{2} \frac{1}{p_{r}^{+}} e^{\bar{\phi}} \partial\left(\bar{\psi}^{+} e^{-i \frac{Q^{2}}{\alpha_{r}} X_{R}^{+}}\right)\left(\bar{z}_{I}\right)\right] \\
& \times c \bar{c} e^{-\phi-\bar{\phi}} V_{r}^{\mathrm{DDF}}\left(Z_{r}, \bar{Z}_{r}\right) .
\end{aligned}
$$

If $z_{I}=z_{I^{(r)}}=z_{I^{\left(r^{\prime}\right)}}$, we get

$$
\begin{aligned}
& \lim _{\epsilon \rightarrow 0}\left[X\left(z_{I}+2 \epsilon\right) \bar{X}\left(\bar{z}_{I}+2 \epsilon\right)\right] \oint_{C_{r,|\epsilon|}} \frac{d \mathbf{z}}{2 \pi i} \mathcal{S}\left(\mathbf{z}, Z_{r}\right) \oint_{\bar{C}_{r,|\epsilon|}} \frac{d \overline{\mathbf{z}}}{2 \pi i} \overline{\mathcal{S}}\left(\overline{\mathbf{z}}, \bar{Z}_{r}\right) c \bar{c} e^{-\phi-\bar{\phi}} V_{r}^{\mathrm{DDF}}\left(Z_{r}, \bar{Z}_{r}\right) \\
& \times \oint_{C_{r^{\prime},|\epsilon|}} \frac{d \mathbf{z}}{2 \pi i} \mathcal{S}\left(\mathbf{z}, Z_{r^{\prime}}\right) \oint_{\bar{C}_{r^{\prime},|\epsilon|}} \frac{d \overline{\mathbf{z}}}{2 \pi i} \overline{\mathcal{S}}\left(\overline{\mathbf{z}}, \bar{Z}_{r^{\prime}}\right) c \bar{c} e^{-\phi-\bar{\phi}} V_{r^{\prime}}^{\mathrm{DDF}}\left(Z_{r^{\prime}}, \bar{Z}_{r^{\prime}}\right) \\
& =\left[\oint_{z_{I}} \frac{d \mathbf{z}}{2 \pi i} \mathcal{S}\left(\mathbf{z}, Z_{r}\right) \oint_{z_{I}} \frac{d \mathbf{z}}{2 \pi i} \mathcal{S}\left(\mathbf{z}, Z_{r^{\prime}}\right) X\left(z_{I}\right)\right. \\
& -\frac{Q^{2}}{2} \frac{1}{p_{r}^{+}}\left\{e^{\phi} \partial\left(\psi^{+} e^{-i \frac{Q^{2}}{\alpha_{r}} X_{L}^{+}}\right)\right\}\left(z_{I}\right) \oint_{z_{I}} \frac{d \mathbf{z}}{2 \pi i} \mathcal{S}\left(\mathbf{z}, Z_{r^{\prime}}\right) \\
& \left.-\oint_{z_{I}} \frac{d \mathbf{z}}{2 \pi i} \mathcal{S}\left(\mathbf{z}, Z_{r}\right) \frac{Q^{2}}{2} \frac{1}{p_{r^{\prime}}^{+}} e^{\phi} \partial\left(\psi^{+} e^{-i \frac{Q^{2}}{\alpha_{r}} X_{L}^{+}}\right)\left(z_{I}\right)\right] \\
& \times\left[\oint_{\bar{z}_{I}} \frac{d \overline{\mathbf{z}}}{2 \pi i} \overline{\mathcal{S}}\left(\overline{\mathbf{z}}, \bar{Z}_{r}\right) \oint_{\bar{z}_{I}} \frac{d \overline{\mathbf{z}}}{2 \pi i} \overline{\mathcal{S}}\left(\overline{\mathbf{z}}, \bar{Z}_{r^{\prime}}\right) \bar{X}\left(\bar{z}_{I}\right)\right. \\
& -\frac{Q^{2}}{2} \frac{1}{p_{r}^{+}}\left\{e^{\bar{\phi}} \partial\left(\bar{\psi}^{+} e^{-i \frac{Q^{2}}{\alpha_{r}} X_{R}^{+}}\right)\right\}\left(\bar{z}_{I}\right) \oint_{\bar{z}_{I}} \frac{d \overline{\mathbf{z}}}{2 \pi i} \overline{\mathcal{S}}\left(\overline{\mathbf{z}}, \bar{Z}_{r^{\prime}}\right)
\end{aligned}
$$




$$
\begin{gathered}
\left.-\oint_{\bar{z}_{I}} \frac{d \overline{\mathbf{z}}}{2 \pi i} \overline{\mathcal{S}}\left(\overline{\mathbf{z}}, \bar{Z}_{r}\right) \frac{Q^{2}}{2} \frac{1}{p_{r^{\prime}}^{+}} e^{\bar{\phi}} \partial\left(\bar{\psi}^{+} e^{-i \frac{Q^{2}}{\alpha_{r^{\prime}}} X_{R}^{+}}\right)\left(\bar{z}_{I}\right)\right] \\
\times c \bar{c} e^{-\phi-\bar{\phi}} V_{r}^{\mathrm{DDF}}\left(Z_{r}, \bar{Z}_{r}\right) c \bar{c} e^{-\phi-\bar{\phi}} V_{r^{\prime}}^{\mathrm{DDF}}\left(Z_{r^{\prime}}, \bar{Z}_{r^{\prime}}\right) .
\end{gathered}
$$

In the generic situation in which $z_{I}, Z_{r}$ are all distinct, ${ }^{3}$ it is not possible for a $z_{I}$ to be equal to $z_{I^{(r)}}$ for more than two $r$ 's, except for the tree-level three point amplitudes. It is possible to derive the formula as (3.14) in such cases. Thus we can see that the limit $\epsilon \rightarrow 0$ in (3.11) is not singular and we denote the result by the naive expression (3.9). Eq. (3.9) is proved in appendix $\mathrm{D}$.

When $Q=0,(3.9)$ becomes

$$
\begin{aligned}
A_{N}^{(g)} \propto & \int[d T][d \alpha][\alpha d \theta] \\
& \times \int\left[d X^{\mu} d \psi^{\mu} d \bar{\psi}^{\mu} d b d \bar{b} d c d \bar{c} d \beta d \bar{\beta} d \gamma d \bar{\gamma}\right]_{g_{z \bar{z}}^{\mathrm{A}}} e^{-S^{\mathrm{tot}}} \\
& \times \prod_{K=1}^{6 g-6+2 N}\left[\oint_{C_{K}} \frac{d z}{\partial \rho} b_{z z}+\varepsilon_{K} \oint_{\bar{C}_{K}} \frac{d \bar{z}}{\bar{\partial} \bar{\rho}} b_{\bar{z} \bar{z}}\right] \prod_{I}\left[X\left(z_{I}\right) \bar{X}\left(\bar{z}_{I}\right)\right] \\
& \times \prod_{r=1}^{N}\left[c \bar{c} e^{-\phi-\bar{\phi}} V_{r}^{\mathrm{DDF}}\left(Z_{r}, \bar{Z}_{r}\right)\right]
\end{aligned}
$$

This expression coincides with the one obtained from the first-quantized formalism putting the PCO's at the interaction points of the light-cone diagram, although this expression suffers from the contact term divergences.

\section{The amplitudes from the first-quantized formalism}

In recent papers [24-26], a way to calculate superstring amplitudes using the PCO's is established. We would like to show that the amplitudes calculated from the light-cone gauge string field theory using the dimensional regularization coincide with those obtained by the method of these papers.

\subsection{The prescription}

In this subsection, we will just briefly explain the prescription given in [26].

In the first-quantized approach using PCO's, an amplitude is expressed by an integral of a correlation function with a fixed number $K$ of PCO insertions over the relevant moduli space $M$ which is assumed to have real dimension $n$. Each point $m \dot{\epsilon} M$ determines a Riemann surface $\Sigma(m)$. Let $Y$ be a fiber bundle over $M$ whose fiber is

$$
\underbrace{\Sigma(m) \times \Sigma(m) \times \cdots \times \Sigma(m)}_{K \text { times }},
$$

in order to describe the insertions of $K$ PCO's, and $X$ be the subspace of $Y$ which is obtained by omitting the bad points where the spurious singularities arise. A point in

\footnotetext{
${ }^{3}$ The situation in which some of $z_{I}, Z_{r}$ coincide can be considered as a limit of these generic situations.
} 


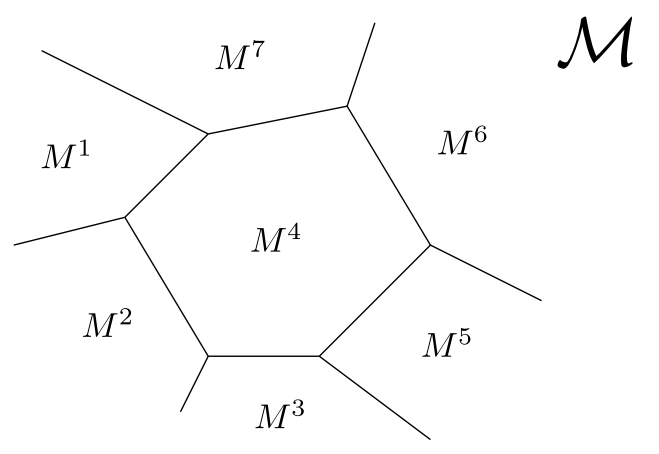

Figure 4. Dual triangulation $\Upsilon$.

$X$ is denoted by $(m ; a)$ with $m \in M, a \in \Sigma(m) \times \Sigma(m) \times \cdots \times \Sigma(m)$ and define a map $\varphi: X \rightarrow M$ which maps $(m ; a)$ to $m$.

If there existed a global section $s$ of $\varphi: X \rightarrow M$, the amplitude would be given by

$$
\int_{M} \omega_{n}(m ; s(m)),
$$

where the integrand is schematically expressed as [24]

$$
\omega_{n}\left(m ; z_{1}, \cdots, z_{K}\right) \propto\left\langle\prod_{i=1}^{K}\left(X\left(z_{i}\right)-\partial \xi\left(z_{i}\right) d z_{i}\right) \prod_{s=1}^{n}\left(\int d^{2} \sigma \frac{\partial\left(\sqrt{g} g_{i j}\right)}{\partial m_{s}} b^{i j} d m_{s}\right) \prod_{r=1}^{N} V_{r}\right\rangle_{n} .
$$

\langle\rangle here denotes the correlation function of the worldsheet theory on $\Sigma(m), V_{1}, \cdots, V_{N}$ are BRST invariant vertex operators, $m_{1}, \cdots, m_{n}$ are the coordinates of $M$ and the subscript $n$ denotes that we should extract the $n$-form part of this expression.

Unfortunately such a global section does not exist in general. If we divide $M$ into patches, we may be able to have a local section on each patch. As was demonstrated in detail in [26], it has been shown that

1. One can pick a dual triangulation $\Upsilon$ of $M$ such that the map $\varphi: X \rightarrow M$ has a local section $s^{\alpha}$ over each of the codimension 0 polyhedron $M^{\alpha}$ in $\Upsilon$ depicted in figure 4 .

2. The amplitude can be given as

$$
\sum_{\alpha} \int_{M^{\alpha}} \omega_{n}\left(m ; s^{\alpha}(m)\right)+A_{\text {vertical }}
$$

where $A_{\text {vertical }}$ is the contribution of the "vertical integration". $A_{\text {vertical }}$ is given as a sum of integrals of correlation functions involving $\xi, X$, antighost insertions and the vertex operators, over $\partial M^{\alpha}$ and their submanifolds which are called the vertical segments.

3. The amplitude thus defined is independent of the choices of $\Upsilon, s^{\alpha}$ and the vertical segments, as long as the bad points are avoided.

4. The amplitude thus defined is gauge invariant. 


\section{2 $Q \rightarrow 0$ limit of the light-cone gauge amplitudes}

As has been shown in [20], the Feynman amplitude (2.21) is well-defined for $Q^{2}>10$ and we can define $A_{N}^{(g)}$ as an analytic function of $Q$, i.e. $A_{N}^{(g)}(Q)$. If the limit $Q \rightarrow 0$ can be taken without encountering any divergences, we will obtain the amplitude without the dilaton background. We would like to show that the limit $Q \rightarrow 0$ is smooth if there are no divergences coming from the boundaries of the moduli space.

As has been shown in section 3, the amplitude (2.21) can be rewritten into a BRST invariant form (3.9). Since the worldsheet theory used in (3.9) consists of the matter superconformal field theory with $\hat{c}=10$ and the superconformal ghosts, the amplitude (3.9) can be recast into the form described in the previous subsection as

$$
A_{N}^{(g)}(Q)=\int_{M} \omega_{n}(m ; s(m)),
$$

where

$$
\begin{aligned}
& \omega_{n}(m ; s(m)) \\
& =d m^{1} \wedge d m^{2} \wedge \cdots \wedge d m^{6 g-6+2 N} \\
& \quad \times \int\left[d X^{\mu} d \psi^{\mu} d \bar{\psi}^{\mu} d b d \bar{b} d c d \bar{c} d \beta d \bar{\beta} d \gamma d \bar{\gamma}\right]_{g_{z \bar{z}}^{\mathrm{A}}} e^{-S^{\mathrm{tot}}} \\
& \quad \times \prod_{K=1}^{6 g-6+2 N}\left[\oint_{C_{K}} \frac{d z}{\partial \rho} b_{z z}+\varepsilon_{K} \oint_{\bar{C}_{K}} \frac{d \bar{z}}{\bar{\partial} \bar{\rho}} b_{\bar{z} \bar{z}}\right] \prod_{I}\left[X\left(z_{I}\right) \bar{X}\left(\bar{z}_{I}\right)\right] \\
& \quad \times \prod_{r=1}^{N}\left[\oint_{I_{I^{(r)}}} \frac{d \mathbf{z}}{2 \pi i} \mathcal{S}\left(\mathbf{z}, Z_{r}\right) \oint_{\bar{z}_{I^{(r)}}} \frac{d \overline{\mathbf{z}}}{2 \pi i} \overline{\mathcal{S}}\left(\overline{\mathbf{z}}, \bar{Z}_{r}\right) c \bar{c} e^{-\phi-\bar{\phi}} V_{r}^{\mathrm{DDF}}\left(Z_{r}, \bar{Z}_{r}\right)\right] .
\end{aligned}
$$

The section $s(m)$ here corresponds to the prescription where the PCO's are located at the interaction points of the light-cone diagram. This expression is well-defined for $Q^{2}>10$ but may suffer from the spurious singularities otherwise.

It is also possible to define amplitudes with the same worldsheet theory and the vertex operators, avoiding the spurious singularities by the Sen-Witten prescription. Namely we can define

$$
A_{N}^{(g) \mathrm{SW}}(Q)=\sum_{\alpha} \int_{M^{\alpha}} \omega_{n}\left(m ; s^{\prime \alpha}(m)\right)+A_{\text {vertical }}
$$

with the local sections $s^{\prime \alpha}(m)$, avoiding the spurious singularities for $Q^{2}<10$. For $Q^{2}>10$,

$$
A_{N}^{(g)}(Q)=A_{N}^{(g) \operatorname{SW}}(Q),
$$

because there are no bad points for $Q^{2}>10$ and the results do not depend on the choice of the local sections. Therefore as an analytic function of $Q, A_{N}^{(g)}(Q)$ coincides with $A_{N}^{(g) \text { SW }}(Q)$ and we obtain

$$
\lim _{Q \rightarrow 0} A_{N}^{(g)}(Q)=A_{N}^{(g) \operatorname{SW}}(0),
$$

if the right hand side exists. The Sen-Witten prescription deals with the spurious singularities and if the superstring amplitudes in question does not suffer from the infrared divergences, $A_{N}^{(g) S W}(0)$ is well-defined. We have shown that the Feynman amplitudes calculated by our method coincide with those obtained by the first-quantized method using the Sen-Witten prescription, as long as we consider infrared safe quantities. 


\section{Conclusions and discussions}

In this paper, we have studied the regularization of the contact term divergences of multiloop scattering amplitudes in the light-cone gauge superstring field theory. We have used the theory in a linear dilaton background $\Phi=-i Q X^{1}$. The divergences of the amplitudes of the theory are thoroughly analyzed in [20]. Since the central charge of this theory is $c=12-12 Q^{2}$, it is possible to shift the central charge to a large negative value by putting $Q^{2}$ large. The multiloop amplitudes involving only the even spin structure with the external lines in the NS-NS sector are indeed regularized by taking $Q^{2}>10$. We have shown that the resultant amplitudes coincide with those of the first-quantized theory through the analytic continuation $Q \rightarrow 0$, without encountering the divergences except those originating from the boundaries of the moduli space. Similarly to the dimensional regularization previously considered [8-11], the amplitudes can be recast into a BRST invariant form of the worldsheet theory in the conformal gauge. This can be achieved by adding the supersymmetric $X^{ \pm} \mathrm{CFT}$ for the longitudinal variables and the superreparametrization ghosts to the worldsheet theory. In the present case, we have constructed BRST invariant worldsheet theory by setting $\frac{d-10}{8}=-Q^{2}$ in the action of the $X^{ \pm}$CFT given in (B.1).

In order to make our regularization scheme complete, we need to deal with the amplitudes for odd spin structure and those with external lines in the Ramond sector. Contrary to the dimensional regularization in which the number of the transverse dimensions $d-2$ is naively shifted, it remains 8 in the present case. This implies that the present procedure does not give rise to the problem pointed out in [11] in constructing the space-time fermions. Furthermore, the light-cone gauge theory in the linear dilaton background used in this paper is much simpler than the theory proposed in [12]. We will investigate this extension elsewhere.

With our prescription, we may be able to describe superstring theory by the simple action with only three string vertices. However there exists subtle points in such a formulation. The action of the light-cone gauge closed superstring field theory possesses only the cubic interactions. While this fact makes the theory simple, the Hamiltonian is unbounded below and thus unstable. The light-cone gauge theory does not contain auxiliary fields and hence the cubic interactions are considered to directly mean the instability of the perturbative vacuum. However, we have to pay attention to the fact that the point where $p^{+}=0$ is not regular in the light-cone gauge formulation and the nonperturbative properties reside there. The stability of the vacuum might not be such a simple problem in the light-cone gauge closed superstring field theory. These facts suggest that it would be desirable to have a gauge invariant string field theory to which our method here is applicable. The conformal gauge expression of the amplitudes given in section 3 may give us a hint about how to construct such a theory. We hope that we will also study these issues elsewhere.

\section{Acknowledgments}

N.I. would like to thank Ted Erler and Ashoke Sen for useful comments. He also would like to thank the organizers of "VIII Workshop on String Field Theory and Related As- 
pects" at São Paulo, especially N. Berkovits, for hospitality. K.M. would like to thank the hospitality of Okayama Institute for Quantum Physics, where part of this work was done. This work was supported in part by Grant-in-Aid for Scientific Research (C) (25400242) and (15K05063) from MEXT.

\section{A Arakelov metric and Arakelov Green's function}

The Arakelov metric $\hat{g}_{z \bar{z}}^{\mathrm{A}}$ and the Arakelov Green's function $G^{\mathrm{A}}(z ; w)$ are defined as follows. Let $\mu_{z \bar{z}}$ be

$$
\mu_{z \bar{z}} \equiv \frac{1}{2 g} \omega(z) \frac{1}{\operatorname{Im} \Omega} \bar{\omega}(\bar{z})
$$

We note that

$$
\int_{\Sigma} d z \wedge d \bar{z} i \mu_{z \bar{z}}=1
$$

which follows from

$$
\int_{\Sigma} \omega_{\mu} \wedge \bar{\omega}_{\nu}=-2 i \operatorname{Im} \Omega_{\mu \nu}
$$

The Arakelov metric on $\Sigma$,

$$
d s_{\AA}^{2}=2 g_{z \bar{z}}^{\mathrm{A}} d z d \bar{z},
$$

is defined so that its scalar curvature $R^{\mathrm{A}} \equiv-2 g^{\mathrm{A} z \bar{z}} \partial \bar{\partial} \ln g_{z \bar{z}}^{\mathrm{A}}$ satisfies

$$
g_{z \bar{z}}^{\mathrm{A}} R^{\mathrm{A}}=-8 \pi(g-1) \mu_{z \bar{z}} .
$$

This condition determines $g_{z \bar{z}}^{\mathrm{A}}$ only up to an overall constant, which will be chosen later.

The Arakelov Green's function $G^{\mathrm{A}}(z, \bar{z} ; w, \bar{w})$ with respect to the Arakelov metric is defined to satisfy ${ }^{4}$

$$
\begin{aligned}
& -\partial_{z} \partial_{\bar{z}} G^{\mathrm{A}}(z, \bar{z} ; w, \bar{w})=2 \pi \delta^{2}(z-w)-2 \pi \mu_{z \bar{z}} \\
& \int_{\Sigma} d z \wedge d \bar{z} i \mu_{z \bar{z}} G^{\mathrm{A}}(z, \bar{z} ; w, \bar{w})=0 .
\end{aligned}
$$

One can obtain a more explicit form of $G^{\mathrm{A}}(z, \bar{z} ; w, \bar{w})$ by solving (A.6) for $G^{\mathrm{A}}(z, \bar{z} ; w, \bar{w})$. Let $F(z, \bar{z} ; w, \bar{w})$ be the $\left(-\frac{1}{2},-\frac{1}{2}\right) \times\left(-\frac{1}{2},-\frac{1}{2}\right)$ form on $\Sigma \times \Sigma$ which satisfies

$$
\partial_{z} \partial_{\bar{z}} \ln F(z, \bar{z} ; w, \bar{w})=2 \pi \delta^{2}(z-w)-2 \pi g \mu_{z \bar{z}}
$$

which can be given by

$$
F(z, \bar{z} ; w, \bar{w})=\exp \left[-2 \pi \operatorname{Im} \int_{w}^{z} \omega \frac{1}{\operatorname{Im} \Omega} \operatorname{Im} \int_{w}^{z} \omega\right]|E(z, w)|^{2} .
$$

Putting (A.7) and (A.5) together, we find that $G^{\mathrm{A}}(z, \bar{z} ; w, \bar{w})$ is given by

$$
G^{\mathrm{A}}(z, \bar{z} ; w, \bar{w})=-\ln F(z, \bar{z} ; w, \bar{w})-\frac{1}{2} \ln \left(2 g_{z \bar{z}}^{\mathrm{A}}\right)-\frac{1}{2} \ln \left(2 g_{w \bar{w}}^{\mathrm{A}}\right),
$$

\footnotetext{
${ }^{4}$ The delta function $\delta^{2}(z-w)$ is normalized by $\int d z \wedge d \bar{z} i \delta^{2}(z-w)=1$.
} 
up to an additive constant independent of $z, \bar{z}$ and $w, \bar{w}$. This possible additive constant can be absorbed into the ambiguity in the overall constant of $g_{z \bar{z}}^{\mathrm{A}}$ mentioned above. It is required that (A.9) holds exactly as it is [34, 37, 38]. This implies that

$$
2 g_{z \bar{z}}^{\mathrm{A}}=\lim _{w \rightarrow z} \exp \left[-G^{\mathrm{A}}(z, \bar{z} ; w, \bar{w})-\ln |z-w|^{2}\right],
$$

and the overall constant of $g_{z \bar{z}}^{\mathrm{A}}$ is, in principle, determined by the second relation in (A.6).

\section{B Supersymmetric $X^{ \pm}$CFT}

The conformal gauge worldsheet theory corresponding to the light-cone gauge superstring theory in noncritical dimensions was studied in [9]. The longitudinal part of it is called the supersymmetric $X^{ \pm}$CFT whose action is given by

$$
S_{\text {super }}^{ \pm}\left[\mathcal{X}^{ \pm} ; \hat{g}_{z \bar{z}}\right]=-\frac{1}{2 \pi} \int d^{2} \mathbf{z}\left(\bar{D} \mathcal{X}^{+} D \mathcal{X}^{-}+\bar{D} \mathcal{X}^{-} D \mathcal{X}^{+}\right)+\frac{d-10}{8} \Gamma_{\text {super }}\left[\mathcal{X}^{+} ; \hat{g}_{z \bar{z}}\right]
$$

Here the supercoordinate $\mathbf{z}$ is given by

$$
\mathbf{z}=(z, \theta),
$$

the superfield $\mathcal{X}^{ \pm}$is defined as

$$
\mathcal{X}^{ \pm}(\mathbf{z}, \overline{\mathbf{z}})=X^{ \pm}(z)+i \theta \psi^{ \pm}(z)+i \bar{\theta} \bar{\psi}^{ \pm}(\bar{z})+\theta \bar{\theta} F^{ \pm},
$$

and

$$
\begin{aligned}
D & \equiv \frac{\partial}{\partial \theta}+\theta \frac{\partial}{\partial z}, \\
\bar{D} & \equiv \frac{\partial}{\partial \bar{\theta}}+\bar{\theta} \frac{\partial}{\partial \bar{z}}, \\
d^{2} \mathbf{z} & \equiv d(\operatorname{Re} z) d(\operatorname{Im} z) d \theta d \bar{\theta} .
\end{aligned}
$$

The interaction term $\Gamma_{\text {super }}$ is given by

$$
\begin{aligned}
\Gamma_{\text {super }}\left[\mathcal{X}^{+} ; \hat{g}_{z \bar{z}}\right] & =-\frac{1}{2 \pi} \int d^{2} \mathbf{z}\left(\bar{D} \Phi D \Phi+\theta \bar{\theta} \hat{g}_{z \bar{z}} \hat{R} \Phi\right) \\
\Phi(\mathbf{z}, \overline{\mathbf{z}}) & =\ln \left(\left(D \Theta^{+}\right)^{2}(\mathbf{z})\left(\bar{D} \bar{\Theta}^{+}\right)^{2}(\overline{\mathbf{z}})\right)-\ln \hat{g}_{z \bar{z}} \\
\Theta^{+}(\mathbf{z}) & =\frac{D \mathcal{X}^{+}}{\left(\partial \mathcal{X}^{+}\right)^{\frac{1}{2}}}(\mathbf{z}),
\end{aligned}
$$

which is the super Liouville action defined for variable $\Phi$ with the background metric $d s^{2}=2 \hat{g}_{z \bar{z}} d z d \bar{z}$. The super energy-momentum tensor $T^{\mathcal{X}^{ \pm}}(\mathbf{z})$ becomes

$$
T^{\mathcal{X}^{ \pm}}(\mathbf{z})=\frac{1}{2} \partial \mathcal{X}^{+} D \mathcal{X}^{-}+\frac{1}{2} \partial \mathcal{X}^{-} D \mathcal{X}^{+}-\frac{d-10}{4} S\left(\mathbf{z}, \mathcal{X}_{\boldsymbol{L}}^{+}\right),
$$

where $S\left(\mathbf{z}, \mathcal{X}_{\boldsymbol{L}}^{+}\right)$denotes the super Schwarzian derivative

$$
S\left(\mathbf{z}, \mathcal{X}_{\boldsymbol{L}}^{+}\right)=\frac{\partial^{2} \Theta^{+}}{D \Theta}-2 \frac{\partial D \Theta^{+} \partial \Theta^{+}}{\left(D \Theta^{+}\right)^{2}}
$$


In the present context, for transverse sector we use the superstring theory in a linear dilaton background instead of the theory in noncritical dimensions. We therefore consider the theory (B.1) with the identification $\frac{d-10}{8}=-Q^{2}$.

The correlation functions to be considered in this theory are defined as

$$
\begin{aligned}
& \left\langle\prod_{r=1}^{N} e^{-i p_{r}^{+} \mathcal{X}^{-}}\left(\mathbf{Z}_{r}, \overline{\mathbf{Z}}_{r}\right) \prod_{s=1}^{M} e^{-i p_{s}^{-} \mathcal{X}^{+}}\left(\mathbf{w}_{s}, \overline{\mathbf{w}}_{s}\right)\right\rangle_{\hat{g}_{z \bar{z}}}^{\mathcal{X}^{ \pm}} \\
& \equiv Z_{\text {super }}^{\mathcal{X}}\left[\hat{g}_{z \bar{z}}\right]^{-2} \\
& \left.\quad \times \int\left[d \mathcal{X}^{+} d \mathcal{X}^{-}\right]_{\hat{g}_{z \bar{z}}} e^{-S_{\text {super }}^{ \pm}\left[\mathcal{X}^{ \pm} ; \hat{g}_{z \bar{z}}\right.}\right] \prod_{r=1}^{N} e^{-i p_{r}^{+} \mathcal{X}^{-}}\left(\mathbf{Z}_{r}, \overline{\mathbf{Z}}_{r}\right) \prod_{s=1}^{M} e^{-i p_{s}^{-} \mathcal{X}^{+}}\left(\mathbf{w}_{s}, \overline{\mathbf{w}}_{s}\right) .
\end{aligned}
$$

Here

$$
Z_{\text {super }}^{\mathcal{X}}\left[\hat{g}_{z \bar{z}}\right]=\int[d \mathcal{X}]_{\hat{g}_{z \bar{z}}} \exp \left[-\frac{1}{2 \pi} \int d^{2} \mathbf{z} \bar{D} \mathcal{X} D \mathcal{X}\right]
$$

and

$$
\begin{aligned}
\mathbf{Z}_{r} & =\left(Z_{r}, \Theta_{r}\right), \\
\mathbf{w}_{s} & =\left(w_{s}, \eta_{s}\right) .
\end{aligned}
$$

In order to discuss these correlation functions, it is convenient to introduce supersymmetric version of $\rho(z)$ in $(2.30)$ which is defined by

$$
\rho_{s}(\mathbf{z})=\rho(z)+\theta f(z),
$$

where

$$
f(z) \equiv-\sum_{r} \alpha_{r} \Theta_{r} S_{\delta}\left(z, Z_{r}\right)
$$

and $S_{\delta}\left(z, z^{\prime}\right)$ is the fermion's Green's function corresponding to the spin structure $\delta$. When all the external lines are in the NS-NS sector and $\delta$ is an even spin structure, $S_{\delta}(z, w)$ is equal to the so-called Szego kernel

$$
S_{\delta}(z, w)=\frac{1}{E(z, w)} \frac{\vartheta[\delta]\left(\int_{w}^{z} \omega \mid \Omega\right)}{\vartheta[\delta](0 \mid \Omega)},
$$

where $\vartheta[\delta](\zeta \mid \Omega)$ denotes the theta function with characteristics $[\delta]=\left[\begin{array}{c}\delta^{\prime} \\ \delta^{\prime \prime}\end{array}\right]$, namely $\delta=$ $\Omega \delta^{\prime}+\delta^{\prime \prime}\left(\delta^{\prime}, \delta^{\prime \prime} \in \mathbb{R}^{g}\right)$, given by

$$
\vartheta[\delta](\zeta \mid \Omega)=\sum_{n \in \mathbb{Z}^{g}} e^{2 \pi i\left[\frac{1}{2}\left(n+\delta^{\prime}\right) \Omega\left(n+\delta^{\prime}\right)+\left(n+\delta^{\prime}\right)\left(\zeta+\delta^{\prime \prime}\right)\right]},
$$

for $\zeta \in \mathbb{C}^{g} /\left(\mathbb{Z}^{g}+\Omega \mathbb{Z}^{g}\right)$. The right hand side of (B.8) can be calculated to be

$$
\begin{aligned}
& \left\langle\prod_{r=1}^{N} e^{-i p_{r}^{+} \mathcal{X}^{-}}\left(\mathbf{Z}_{r}, \overline{\mathbf{Z}}_{r}\right) \prod_{s=1}^{M} e^{-i p_{s}^{-} \mathcal{X}^{+}}\left(\mathbf{w}_{s}, \overline{\mathbf{w}}_{s}\right)\right\rangle_{\hat{g}_{z \bar{z}}}^{\mathcal{X}^{ \pm}} \\
& =(2 \pi)^{2} \delta\left(\sum_{s} p_{s}^{-}\right) \delta\left(\sum_{r} p_{r}^{+}\right) \prod_{s} e^{-p_{s}^{-} \frac{\rho_{s}+\bar{\rho} s}{2}}\left(\mathbf{w}_{s}, \overline{\mathbf{w}}_{s}\right) e^{-\frac{d-10}{8} \Gamma_{\text {super }}\left[\rho_{s}+\bar{\rho}_{s} ; \hat{g}_{z \bar{z}}\right]},
\end{aligned}
$$


where

$$
\exp \left(-\Gamma_{\text {super }}\left[\rho_{s}+\bar{\rho}_{s} ; \hat{g}_{z \bar{z}}\right]\right)=\exp \left(-\frac{1}{2} \Gamma\left[\sigma ; \hat{g}_{z \bar{z}}\right]-\sum_{r} \Delta \Gamma_{r}-\sum_{I} \Delta \Gamma_{I}\right)
$$

with

$$
\begin{aligned}
-\Delta \Gamma_{r}= & \frac{1}{2 \alpha_{r}} \frac{\partial f f}{\partial^{2} \rho}\left(z_{I^{(r)}}\right)+\text { c.c. }, \\
-\Delta \Gamma_{I}= & \left\{-\left(\frac{5}{12} \frac{\partial^{4} \rho}{\left(\partial^{2} \rho\right)^{3}}-\frac{3}{4} \frac{\left(\partial^{3} \rho\right)^{2}}{\left(\partial^{2} \rho\right)^{4}}\right) \partial f f+\frac{2}{3} \frac{\partial^{3} f f}{\left(\partial^{2} \rho\right)^{2}}-\frac{\partial^{3} \rho}{\left(\partial^{2} \rho\right)^{3}} \partial^{2} f f\right. \\
& \left.-\frac{1}{12} \frac{\partial^{3} f \partial^{2} f \partial f f}{\left(\partial^{2} \rho\right)^{4}}\right\}\left(z_{I}\right)+\text { c.c. },
\end{aligned}
$$

and $\Gamma\left[\sigma ; \hat{g}_{z \bar{z}}\right]$ given in (2.31). From (B.15), it is possible to deduce all the correlation functions of the $X^{ \pm}$CFT.

The correlation functions of fermions. From (B.15), one can derive a formula of the correlation functions of fermions $\psi^{ \pm}, \bar{\psi}^{ \pm}$, which is useful in appendix D. (B.15) can be rewritten as $^{5}$

$$
\begin{aligned}
& \left\langle\prod_{r=1}^{N} e^{-i p_{r}^{+} X^{-}+p_{r}^{+}\left(\Theta_{r} \psi^{-}+\bar{\Theta}_{r} \bar{\psi}^{-}\right)}\left(Z_{r}, \bar{Z}_{r}\right) \prod_{s=1}^{M} e^{-i p_{s}^{-} X^{+}+p_{s}^{-}\left(\eta_{s} \psi^{+}+\bar{\eta}_{s} \bar{\psi}^{+}\right)}\left(w_{s}, \bar{w}_{s}\right)\right\rangle_{\hat{g}_{z \bar{z}}}^{\mathcal{X}^{ \pm}} \\
& =(2 \pi)^{2} \delta\left(\sum_{s} p_{s}^{-}\right) \delta\left(\sum_{r} p_{r}^{+}\right) \prod_{s} e^{-p_{s}^{-\frac{1}{2}(\rho+\bar{\rho})}\left(w_{s}, \bar{w}_{s}\right) e^{-\frac{d-10}{16} \Gamma}} \\
& \quad \times \prod_{s} e^{-p_{s}^{-} \frac{1}{2}\left(\eta_{s} f\left(w_{s}\right)+\bar{\eta}_{s} \bar{f}\left(\bar{w}_{s}\right)\right)} e^{-\frac{d-10}{8}\left(\sum_{r} \Delta \Gamma_{r}+\sum_{I} \Delta \Gamma_{I}\right)}
\end{aligned}
$$

The fermionic contribution of this correlation function can be expressed as

$$
\begin{aligned}
\prod_{s} e^{-p_{s}^{-} \frac{1}{2}\left(\eta_{s} f\left(w_{s}\right)+\bar{\eta}_{s} \bar{f}\left(\bar{w}_{s}\right)\right)} e^{-\frac{d-10}{8}\left(\sum_{r} \Delta \Gamma_{r}+\sum_{I} \Delta \Gamma_{I}\right)} \\
=\left(Z^{\psi}\left[\hat{g}_{z \bar{z}}\right]\right)^{-2} \int\left[d \psi^{+} d \psi^{-} d \bar{\psi}^{+} d \bar{\psi}^{-}\right] e^{\frac{1}{\pi} \int d^{2} z\left(\psi^{-} \bar{\partial} \psi^{+}+\bar{\psi}^{-} \partial \bar{\psi}^{+}\right)-S_{\mathrm{int}}} \\
\quad \times \prod_{r=1}^{N} e^{p_{r}^{+}\left(\Theta_{r} \psi^{-}+\bar{\Theta}_{r} \bar{\psi}^{-}\right)}\left(Z_{r}, \bar{Z}_{r}\right) \prod_{s=1}^{M} e^{p_{s}^{-}\left(\eta_{s} \psi^{+}+\bar{\eta}_{s} \bar{\psi}^{+}\right)}\left(w_{s}, \bar{w}_{s}\right),
\end{aligned}
$$

\footnotetext{
${ }^{5}$ From the correlation functions, we can see that $F^{+}$and $F^{-}$can be put equal to zero.
} 
where

$$
\begin{aligned}
S_{\mathrm{int}}=\frac{d-10}{8}[ & -\sum_{r} \frac{2}{\alpha_{r}} \frac{\partial \psi^{+} \psi^{+}}{\partial^{2} \rho}\left(z_{I^{(r)}}\right) \\
& +\sum_{I}\left\{\left(\frac{5}{3} \frac{\partial^{4} \rho}{\left(\partial^{2} \rho\right)^{3}}-3 \frac{\left(\partial^{3} \rho\right)^{2}}{\left(\partial^{2} \rho\right)^{4}}\right) \partial \psi^{+} \psi^{+}-\frac{8}{3} \frac{\partial^{3} \psi^{+} \psi^{+}}{\left(\partial^{2} \rho\right)^{2}}\right. \\
& \left.\quad+\frac{4 \partial^{3} \rho}{\left(\partial^{2} \rho\right)^{3}} \partial^{2} \psi^{+} \psi^{+}+\frac{4}{3} \frac{\partial^{3} \psi^{+} \partial^{2} \psi^{+} \partial \psi^{+} \psi^{+}}{\left(\partial^{2} \rho\right)^{4}}\right\}\left(z_{I}\right) \\
& + \text { c.c. }] .
\end{aligned}
$$

Substituting (B.19) into (B.18) and differentiating with respect to $\Theta_{r}, \bar{\Theta}_{r}, \eta_{s}, \bar{\eta}_{s}$, we obtain the following identity

$$
\begin{gathered}
\left\langle\prod_{r=1}^{N} e^{-i p_{r}^{+} X^{-}}\left(Z_{r}, \bar{Z}_{r}\right) \prod_{s=1}^{M} e^{-i p_{s}^{-} X^{+}}\left(w_{s}, \bar{w}_{s}\right) \psi^{+}\left(u_{1}\right) \cdots \psi^{+}\left(u_{n}\right) \psi^{-}\left(v_{1}\right) \cdots \psi^{-}\left(v_{m}\right)\right. \\
\left.\times \bar{\psi}^{+}\left(\tilde{u}_{1}\right) \cdots \bar{\psi}^{+}\left(\tilde{u}_{n}\right) \bar{\psi}^{-}\left(\tilde{v}_{1}\right) \cdots \bar{\psi}^{-}\left(\tilde{v}_{m}\right)\right\rangle_{\hat{g}_{z \bar{z}}}^{\mathcal{X}^{ \pm}} \\
=(2 \pi)^{2} \delta\left(\sum_{s} p_{s}^{-}\right) \delta\left(\sum_{r} p_{r}^{+}\right) \prod_{s} e^{-p_{s}^{-\frac{1}{2}(\rho+\bar{\rho})}\left(w_{s}, \bar{w}_{s}\right) e^{-\frac{d-10}{16} \Gamma}} \\
\times\left(Z^{\psi}\left[\hat{g}_{z \bar{z}}\right]\right)^{-2} \int\left[d \psi^{+} d \psi^{-} d \bar{\psi}^{+} d \bar{\psi}^{-}\right] e^{\frac{1}{\pi} \int d^{2} z\left(\psi^{-} \bar{\partial} \psi^{+}+\bar{\psi}^{-} \partial \bar{\psi}^{+}\right)-S_{\mathrm{int}}} \\
\times \psi^{+}\left(u_{1}\right) \cdots \psi^{+}\left(u_{n}\right) \psi^{-}\left(v_{1}\right) \cdots \psi^{-}\left(v_{m}\right) \\
\times \bar{\psi}^{+}\left(\tilde{u}_{1}\right) \cdots \bar{\psi}^{+}\left(\tilde{u}_{n}\right) \bar{\psi}^{-}\left(\tilde{v}_{1}\right) \cdots \bar{\psi}^{-}\left(\tilde{v}_{m}\right) \cdot \quad(\mathrm{B} .21)
\end{gathered}
$$

Namely, the fermionic part of the correlation functions of supersymmetric $X^{ \pm}$CFT coincide with those of the theory with interaction $S_{\text {int }}$ which is localized at the interaction points $z_{I}$.

\section{Ghost systems on higher genus Riemann surfaces}

In this appendix, we would like to show some identities which are crucial in deriving the conformal gauge expression of the light-cone gauge amplitudes in section 3 .

Let us consider the conformal field theory with the action

$$
\frac{1}{\pi} \int d z \wedge d \bar{z} \sqrt{g}\left(b \nabla^{z} c+\bar{b} \nabla^{\bar{z}} \bar{c}\right)
$$

where the fields $b, c$ are with conformal weight $(\lambda, 0),(1-\lambda, 0)$ and $\bar{b}, \bar{c}$ are their antiholomorphic counterparts with conformal weight $(0, \lambda),(0,1-\lambda)$. Here we consider the case either $\lambda \in \mathbb{Z}$ or $\lambda \in \mathbb{Z}+\frac{1}{2}$. The fields can be either Grassmann odd or even accordingly. We define $\epsilon= \pm 1$ to be

$$
\epsilon=\left\{\begin{array}{ll}
1 & \text { if } b, c \text { are Grassmann odd } \\
-1 & \text { if } b, c \text { are Grassmann even }
\end{array} .\right.
$$


There exist local operators $e^{q \phi}(z, \bar{z})\left(q \in \frac{\mathbb{Z}}{2}\right)$, which satisfy

$$
\begin{aligned}
& b(z) e^{q \phi}(w, \bar{w}) \sim(z-w)^{-\epsilon q}, \\
& c(z) e^{q \phi}(w, \bar{w}) \sim(z-w)^{\epsilon q}, \\
& \bar{b}(\bar{z}) e^{q \phi}(w, \bar{w}) \sim(\bar{z}-\bar{w})^{-\epsilon q}, \\
& \bar{c}(\bar{z}) e^{q \phi}(w, \bar{w}) \sim(\bar{z}-\bar{w})^{\epsilon q} .
\end{aligned}
$$

We would like to discuss the correlation functions of the form

$$
\left\langle\prod_{i} e^{\epsilon q_{i} \phi}\left(z_{i}, \bar{z}_{i}\right)\right\rangle
$$

on a genus $g$ Riemann surface. $q_{i}$ should satisfy

$$
\sum_{i} q_{i}=-(2 \lambda-1)(g-1) .
$$

When $\lambda \in \mathbb{Z}+\frac{1}{2}$, the correlation function we consider here is the one corresponding to a spin structure $\left[\begin{array}{c}\alpha^{\prime} \\ \alpha^{\prime \prime}\end{array}\right]$. Namely, the fields $b(z), c(z), \bar{b}(\bar{z}), \bar{c}(\bar{z})$ transform as

$$
\begin{aligned}
& c(z) \rightarrow e^{2 \pi i \alpha_{\mu}^{\prime}} c(z), \\
& b(z) \rightarrow e^{2 \pi i \alpha_{\mu}^{\prime}} b(z), \\
& \bar{c}(\bar{z}) \rightarrow e^{2 \pi i \alpha_{\mu}^{\prime}} \bar{c}(\bar{z}), \\
& \bar{b}(\bar{z}) \rightarrow e^{2 \pi i \alpha_{\mu}^{\prime}} \bar{b}(\bar{z}),
\end{aligned}
$$

if $z$ is moved around the $a_{\mu}(\mu=1, \cdots, g)$ cycle once, and they transform as

$$
\begin{aligned}
& c(z) \rightarrow e^{2 \pi i \alpha_{\mu}^{\prime \prime}} c(z), \\
& b(z) \rightarrow e^{2 \pi i \alpha_{\mu}^{\prime \prime}} b(z), \\
& \bar{c}(\bar{z}) \rightarrow e^{2 \pi i \alpha_{\mu}^{\prime \prime}} \bar{c}(\bar{z}), \\
& \bar{b}(\bar{z}) \rightarrow e^{2 \pi i \alpha_{\mu}^{\prime \prime} \bar{b}(\bar{z}),}
\end{aligned}
$$

if $z$ is moved around the $b_{\mu}$ cycle once. We take $\alpha^{\prime}=\alpha^{\prime \prime}=0$ for $\lambda \in \mathbb{Z}$.

Taking the metric on the worldsheet to be the Arakelov metric $g_{z_{i} \bar{z}_{i}}^{A}$, the correlation function $\left\langle\prod_{i} e^{\epsilon q_{i} \phi}\left(z_{i}, \bar{z}_{i}\right)\right\rangle$ is evaluated in [35] to be

$$
\left\langle\prod_{i} e^{\epsilon q_{i} \phi}\left(z_{i}, \bar{z}_{i}\right)\right\rangle \prod_{i}\left(g_{z_{i} \bar{z}_{i}}^{A}\right)^{d\left(q_{i}\right)} \propto\left[\left(\frac{\operatorname{det}^{\prime}\left(-g^{\mathrm{A} z \bar{z}} \partial_{z} \partial_{\bar{z}}\right)}{\operatorname{det} \operatorname{Im} \Omega \int d^{2} z \sqrt{g^{\mathrm{A}}}}\right)^{-\frac{1}{2}}\left|\vartheta\left[\begin{array}{l}
a \\
b
\end{array}\right](0 \mid \Omega)\right|^{2} \prod_{i>j} e^{-q_{i} q_{j} G^{\mathrm{A}}\left(z_{i}, z_{j}\right)}\right]_{(\mathrm{C} .8)}^{\epsilon}
$$

where

$$
d(q)=\frac{1}{2} \epsilon q(q+1-2 \lambda),
$$

and the characteristics $\left[\begin{array}{l}a \\ b\end{array}\right]$ is defined so that

$$
(\Omega a+b)_{\nu}=-\sum_{i} q_{i} \int_{P_{0}}^{z_{i}} \omega_{\nu}-(2 \lambda-1) \int_{P_{0}}^{\triangle} \omega_{\nu}+\left(\Omega \alpha^{\prime}+\alpha^{\prime \prime}\right)_{\nu} .
$$

Here $\Delta$ is the Riemann class, which is related to the canonical divisor $K$ of the Riemann surface by $2 \triangle=K$. 


\section{C.1 A formula for the superghosts}

Since the correlation function (C.8) is left-right symmetric, we need some more work to get a formula which is useful for superghosts. We will present it in a form factorized in chiral and anti-chiral parts. By doing so, it is possible to get the correlation functions which are not left-right symmetric with respect to the choice of local operators $e^{q \phi}$ and spin structure.

Substituting (A.8), (A.9) and

$$
\vartheta\left[\begin{array}{l}
a \\
b
\end{array}\right](0 \mid \Omega)=e^{\pi i a \Omega a+2 \pi i a b-\pi i \alpha^{\prime} \Omega \alpha^{\prime}-2 \pi i \alpha^{\prime}\left(e+\alpha^{\prime \prime}\right)} \vartheta\left[\begin{array}{c}
\alpha^{\prime} \\
\alpha^{\prime \prime}
\end{array}\right](e \mid \Omega),
$$

with

$$
e_{\nu}=-\sum_{i} q_{i} \int_{P_{0}}^{z_{i}} \omega_{\nu}-(2 \lambda-1) \int_{P_{0}}^{\triangle} \omega_{\nu}
$$

into the right hand side of (C.8), we obtain for $g \neq 1$

$$
\begin{aligned}
& \left\langle\prod_{i} e^{\epsilon q_{i} \phi}\left(z_{i}, \bar{z}_{i}\right)\right\rangle \\
& =\left[\left(\frac{\operatorname{det}^{\prime}\left(-g^{\mathrm{A} z \bar{z}} \partial_{z} \partial_{\bar{z}}\right)}{\operatorname{det} \operatorname{Im} \Omega \int d^{2} z \sqrt{g^{\mathrm{A}}}}\right)^{-\frac{1}{2}}\left|\vartheta\left[\begin{array}{c}
\alpha^{\prime} \\
\alpha^{\prime \prime}
\end{array}\right](e \mid \Omega)\right|^{2} \prod_{i>j}\left|E\left(z_{i}, z_{j}\right)\right|^{2 q_{i} q_{j}}\right. \\
& \left.\times \prod_{i}\left(\left(g_{z_{i} \bar{z}_{i}}^{A}\right)^{\frac{g}{2}} \exp \left[-\frac{2 \pi}{g-1} \operatorname{Im} \int_{(g-1) z_{i}}^{\triangle} \omega(\operatorname{Im} \Omega)^{-1} \operatorname{Im} \int_{(g-1) z_{i}}^{\triangle} \omega\right]\right)^{-q_{i}(2 \lambda-1)}\right]^{\epsilon} .
\end{aligned}
$$

Using (A.5), we can see

$$
\partial_{z} \partial_{\bar{z}} \ln \left(\left(g_{z \bar{z}}^{A}\right)^{\frac{g}{2}} \exp \left[-\frac{2 \pi}{g-1} \operatorname{Im} \int_{(g-1) z}^{\triangle} \omega(\operatorname{Im} \Omega)^{-1} \operatorname{Im} \int_{(g-1) z}^{\triangle} \omega\right]\right)=0 .
$$

Therefore there exists a holomorphic $\frac{g}{2}$ form $\sigma(z)$ such that

$$
\left(g_{z \bar{z}}^{A}\right)^{\frac{g}{2}} \exp \left[-\frac{2 \pi}{g-1} \operatorname{Im} \int_{(g-1) z}^{\triangle} \omega(\operatorname{Im} \Omega)^{-1} \operatorname{Im} \int_{(g-1) z}^{\triangle} \omega\right]=|\sigma(z)|^{2} e^{\frac{3}{g-1} S},
$$

where $S$ is independent of $z \cdot \sigma(z)$ has no zeros or poles, and it should transform as

$$
\sigma(z) \rightarrow e^{-2 \pi i \int_{(g-1) z}^{\Delta} \omega_{\mu}+\pi i(g-1) \Omega_{\mu \mu}} \sigma(z),
$$

when $z$ is moved around the $b_{\mu}$ cycle once, and invariant when $z$ is moved around the $a_{\mu}$ cycles. These properties fix $\sigma(z)$ and it should coincide with the $\sigma(z)$ in [30,33] up to a multiplicative factor. Substituting (C.15) into (C.13), we obtain

$$
\begin{aligned}
& \left\langle\prod_{i} e^{\epsilon q_{i} \phi}\left(z_{i}, \bar{z}_{i}\right)\right\rangle \\
& =\left[\left(\frac{\operatorname{det}^{\prime}\left(-g^{\mathrm{A} z \bar{z}} \partial_{z} \partial_{\bar{z}}\right)}{\operatorname{det} \operatorname{Im} \Omega \int d^{2} z \sqrt{g^{\mathrm{A}}}}\right)^{-\frac{1}{2}}\left|\vartheta\left[\begin{array}{c}
\alpha^{\prime} \\
\alpha^{\prime \prime}
\end{array}\right](e \mid \Omega) \prod_{i>j} E\left(z_{i}, z_{j}\right)^{q_{i} q_{j}} \prod_{i} \sigma\left(z_{i}\right)^{-q_{i}(2 \lambda-1)}\right|^{2}\right]^{\epsilon} \\
& \quad \times e^{3(2 \lambda-1)^{2} \epsilon S}
\end{aligned}
$$


For $g=1$, instead of (C.13) we get

$$
\begin{aligned}
& \left\langle\prod_{i} e^{\epsilon q_{i} \phi}\left(z_{i}, \bar{z}_{i}\right)\right\rangle \\
& =\left[\left(\frac{\operatorname{det}^{\prime}\left(-g^{\mathrm{A} z \bar{z}} \partial_{z} \partial_{\bar{z}}\right)}{\operatorname{det} \operatorname{Im} \Omega \int d^{2} z \sqrt{g^{\mathrm{A}}}}\right)^{-\frac{1}{2}}\left|\vartheta\left[\begin{array}{c}
\alpha^{\prime} \\
\alpha^{\prime \prime}
\end{array}\right](e \mid \Omega)\right|^{2} \prod_{i>j}\left|E\left(z_{i}, z_{j}\right)\right|^{2 q_{i} q_{j}}\right]^{\epsilon} \\
& \times \prod_{i}\left(\left(g_{z_{i} \bar{z}_{i}}^{A}\right)^{\frac{1}{2}} \exp \left[4 \pi \operatorname{Im} \int_{P_{0}}^{z_{i}} \omega(\operatorname{Im} \Omega)^{-1} \operatorname{Im} \int_{P_{0}}^{\triangle} \omega\right]\right)^{-q_{i} \epsilon(2 \lambda-1)} \\
& \times \exp \left[-2 \pi(2 \lambda-1)^{2} \epsilon \operatorname{Im} \int_{P_{0}}^{\triangle} \omega(\operatorname{Im} \Omega)^{-1} \operatorname{Im} \int_{P_{0}}^{\triangle} \omega\right] .
\end{aligned}
$$

Since

$$
\begin{aligned}
-2 \partial \bar{\partial} \ln g_{z \bar{z}}^{\mathrm{A}} & =-8 \pi(g-1) \mu_{z \bar{z}}=0, \\
\sum_{i} q_{i} & =-(2 \lambda-1)(g-1)=0,
\end{aligned}
$$

putting

$$
\begin{aligned}
\left(g_{z \bar{z}}^{A}\right)^{\frac{g}{2}} \exp \left[4 \pi \operatorname{Im} \int_{P_{0}}^{z} \omega(\operatorname{Im} \Omega)^{-1} \operatorname{Im} \int_{P_{0}}^{\triangle} \omega\right] & \equiv|\sigma(z)|^{2} e^{A}, \\
\exp \left[-2 \pi(2 \lambda-1)^{2} \operatorname{Im} \int_{P_{0}}^{\triangle} \omega(\operatorname{Im} \Omega)^{-1} \operatorname{Im} \int_{P_{0}}^{\triangle} \omega\right] & \equiv e^{3(2 \lambda-1)^{2} S},
\end{aligned}
$$

with $S, A$ independent of $z$, we get (C.17).

In (C.17), the correlation function factorizes into the left and right parts except for the determinant factor and $e^{-3(2 \lambda-1)^{2} S}$. The determinant factor can also be recast into a factorized form as follows. Let us consider the $b c$-system with $\lambda=1, \epsilon=1$. For arbitrary $R, z_{i}(i=1, \ldots, g),($ C.17) implies

$$
\begin{aligned}
& \left\langle e^{\phi}(R, \bar{R}) \prod_{i=1}^{g} e^{-\phi}\left(z_{i}, \bar{z}_{i}\right)\right\rangle=\frac{\operatorname{det}^{\prime}\left(-g^{\mathrm{A} z \bar{z}} \partial_{z} \partial_{\bar{z}}\right)}{\operatorname{det} \operatorname{Im} \Omega \int d^{2} z \sqrt{g^{\mathrm{A}}}}\left|\operatorname{det} \omega_{\nu z_{i}}\right|^{2} \\
& =\left(\frac{\operatorname{det}^{\prime}\left(-g^{\mathrm{A} z \bar{z}} \partial_{z} \partial_{\bar{z}}\right)}{\operatorname{det} \operatorname{Im} \Omega \int d^{2} z \sqrt{g^{\mathrm{A}}}}\right)^{-\frac{1}{2}}\left|\vartheta\left[\begin{array}{l}
0 \\
0
\end{array}\right](e \mid \Omega) \frac{\prod_{i>j} E\left(z_{i}, z_{j}\right)}{\prod_{i} E\left(z_{i}, R\right)} \frac{\prod_{i} \sigma\left(z_{i}\right)}{\sigma(R)}\right|^{2} e^{3 S},
\end{aligned}
$$

and we get

$$
\left(\frac{\operatorname{det}^{\prime}\left(-g^{\mathrm{A} z \bar{z}} \partial_{z} \partial_{\bar{z}}\right)}{\operatorname{det} \operatorname{Im} \Omega \int d^{2} z \sqrt{g^{\mathrm{A}}}}\right)^{-\frac{1}{2}}=\left|\left(\frac{\prod_{i} E\left(z_{i}, R\right) \sigma(R) \operatorname{det} \omega_{\nu z_{i}}}{\vartheta\left[\begin{array}{l}
0 \\
0
\end{array}\right](e \mid \Omega) \prod_{i>j} E\left(z_{i}, z_{j}\right) \prod_{i} \sigma\left(z_{i}\right)}\right)^{\frac{1}{3}}\right|^{2} e^{-S} .
$$


Therefore (C.17) can be rewritten as

$$
\begin{aligned}
\left\langle\prod_{i} e^{\epsilon q_{i} \phi}\left(z_{i}, \bar{z}_{i}\right)\right\rangle=\mid & \left.\left(\frac{\prod_{i} E\left(z_{i}, R\right) \sigma(R) \operatorname{det} \omega_{\nu z_{i}}}{\vartheta\left[\begin{array}{l}
0 \\
0
\end{array}\right](e \mid \Omega) \prod_{i>j} E\left(z_{i}, z_{j}\right) \prod_{i} \sigma\left(z_{i}\right)}\right)^{\frac{1}{3}}\right|^{2 \epsilon} \\
& \times\left|\vartheta\left[\begin{array}{c}
\alpha^{\prime} \\
\alpha^{\prime \prime}
\end{array}\right](e \mid \Omega) \prod_{i>j} E\left(z_{i}, z_{j}\right)^{q_{i} q_{j}} \prod_{i} \sigma\left(z_{i}\right)^{-q_{i}(2 \lambda-1)}\right|^{2 \epsilon} \\
& \times e^{-\epsilon\left(-3(2 \lambda-1)^{2}+1\right) S} .
\end{aligned}
$$

$\epsilon\left(-3(2 \lambda-1)^{2}+1\right)$ coincides with the central charge of the $b c$-system and $e^{-\epsilon\left(-3(2 \lambda-1)^{2}+1\right) S}$ can be identified with the holomorphic anomaly. One can construct the vacuum amplitude of critical string theory, combining these correlation functions. With vanishing central charge, the vacuum amplitude completely factorizes into the holomorphic and antiholomorphic parts.

The holomorphic and antiholomorphic parts of the correlation function can be read off from (C.17) and we can get the correlation functions which are not left-right symmetric by combining the holomorphic and antiholomorphic parts. For example, the partition function of a free Dirac fermion with spin structure $\left[\alpha_{L}\right]$ for left and $\left[\alpha_{R}\right]$ for right can be given by

$$
\left(Z^{\psi}\left[g_{z \bar{z}}^{\mathrm{A}}\right]\right)^{2}=\left(\frac{\operatorname{det}^{\prime}\left(-g^{\mathrm{A} z \bar{z}} \partial_{z} \partial_{\bar{z}}\right)}{\operatorname{det} \operatorname{Im} \Omega \int d^{2} z \sqrt{g^{\mathrm{A}}}}\right)^{-\frac{1}{2}} \vartheta\left[\alpha_{L}\right](0 \mid \Omega) \vartheta\left[\alpha_{R}\right](0 \mid \Omega)^{*}
$$

The correlation function

$$
\int[d \beta d \bar{\beta} d \gamma d \bar{\gamma}] e^{-S_{\beta \gamma}} \prod_{I}\left|\delta\left(\beta\left(z_{I}\right)\right)\right|^{2} \prod_{r}\left|\delta\left(\gamma\left(Z_{r}\right)\right)\right|^{2}
$$

of the superreparametrization ghost with spin structure $\left[\alpha_{L}\right]$ for left and $\left[\alpha_{R}\right]$ for right can be evaluated to be

$$
\begin{aligned}
\left(\frac{\operatorname{det}^{\prime}\left(-g^{\mathrm{A} z \bar{z}} \partial_{z} \partial_{\bar{z}}\right)}{\operatorname{det} \operatorname{Im} \Omega \int d^{2} z \sqrt{g^{\mathrm{A}}}}\right)^{\frac{1}{2}}\left(\vartheta\left[\alpha_{L}\right]\left(-\sum_{r} \int_{P_{0}}^{Z_{r}} \omega+\sum_{I} \int_{P_{0}}^{z_{I}} \omega-2 \int_{P_{0}}^{\triangle} \omega \mid \Omega\right)\right)^{-1} \\
\times\left(\vartheta\left[\alpha_{R}\right]\left(-\sum_{r} \int_{P_{0}}^{Z_{r}} \omega+\sum_{I} \int_{P_{0}}^{z_{I}} \omega-2 \int_{P_{0}}^{\triangle} \omega \mid \Omega\right)^{*}\right)^{-1} \\
\times\left|\frac{\prod_{I, r} E\left(z_{I}, Z_{r}\right)}{\prod_{I>J} E\left(z_{I}, z_{J}\right) \prod_{r>s} E\left(Z_{r}, Z_{s}\right)} \frac{\prod_{r} \sigma\left(Z_{r}\right)^{2}}{\prod_{I} \sigma\left(z_{I}\right)^{2}}\right|^{2} e^{-12 S}
\end{aligned}
$$

Since $Z_{r}(r=1, \ldots N)$ and $z_{I}(I=1, \ldots, 2 g-2+N)$ are the zeros and the poles of the meromorphic one-form $\partial \rho(z) d z$ respectively,

$$
-\sum_{r} Z_{r}+\sum_{I} z_{I}=K=2 \triangle
$$


holds in the divisor sense. Therefore we obtain

$$
\begin{aligned}
& \left(Z^{\psi}\left[g_{z \bar{z}}^{\mathrm{A}}\right]\right)^{2} \int[d \beta d \gamma] e^{-S_{\beta \gamma}} \prod_{I}\left|\delta\left(\beta\left(z_{I}\right)\right)\right|^{2} \prod_{r}\left|\delta\left(\gamma\left(Z_{r}\right)\right)\right|^{2} \\
& \quad=\left|\frac{\prod_{I, r} E\left(z_{I}, Z_{r}\right)}{\prod_{I>J} E\left(z_{I}, z_{J}\right) \prod_{r>s} E\left(Z_{r}, Z_{s}\right)} \frac{\prod_{r} \sigma\left(Z_{r}\right)^{2}}{\prod_{I} \sigma\left(z_{I}\right)^{2}}\right|^{2} e^{-12 S} .
\end{aligned}
$$

On the other hand, (A.8), (A.9) and (C.15) imply

$$
\begin{aligned}
\left|\frac{\prod_{I, r} E\left(z_{I}, Z_{r}\right)}{\prod_{I>J} E\left(z_{I}, z_{J}\right) \prod_{r>s} E\left(Z_{r}, Z_{s}\right)} \frac{\prod_{r} \sigma\left(Z_{r}\right)^{2}}{\prod_{I} \sigma\left(z_{I}\right)^{2}}\right|^{2} e^{-12 S} \\
=\exp \left[\sum_{I<J} G^{\mathrm{A}}\left(z_{I} ; z_{J}\right)+\sum_{r<s} G^{\mathrm{A}}\left(Z_{r} ; Z_{s}\right)-\sum_{I, r} G^{\mathrm{A}}\left(z_{I} ; Z_{r}\right)\right] \\
\times \prod_{I}\left(2 g_{z_{I} \bar{z}_{I}}^{\mathrm{A}}\right)^{-\frac{3}{2}} \prod_{r}\left(2 g_{Z_{r} \bar{Z}_{r}}^{\mathrm{A}}\right)^{\frac{1}{2}}
\end{aligned}
$$

and from (2.31) we get

$$
\begin{gathered}
\prod_{r=1}^{N} e^{-\operatorname{Re} \bar{N}_{00}^{r r}} \prod_{I}\left|\partial^{2} \rho\left(z_{I}\right)\right|^{-\frac{3}{2}} e^{\frac{1}{2} \Gamma\left[g_{z \bar{z}}^{\mathrm{A}}, \rho+\bar{\rho}\right]}\left(Z^{\psi}\left[g_{z \bar{z}}^{\mathrm{A}}\right]\right)^{-2} \\
\quad=\int[d \beta d \gamma] e^{-S_{\beta \gamma}} \prod_{I}\left|\delta\left(\beta\left(z_{I}\right)\right)\right|^{2} \prod_{r}\left|\delta\left(\gamma\left(Z_{r}\right)\right)\right|^{2}
\end{gathered}
$$

This identity is crucial for deriving the BRST invariant expression of the superstring amplitudes.

\section{C.2 A formula for the reparametrization ghosts}

In [13] it was shown that the following identity holds:

$$
\begin{aligned}
& \prod_{r=1}^{N}\left(\alpha_{r} e^{2 \operatorname{Re} \bar{N}_{00}^{r r}}\right) e^{-\Gamma\left[g_{z \bar{z}}^{\mathrm{A}}, \rho+\bar{\rho}\right]}\left(Z^{X}\left[g_{z \bar{z}}^{\mathrm{A}}\right]\right)^{-2} \\
& =\text { const. } \int[d b d \bar{b} d c d \bar{c}]_{g_{z \bar{z}}^{\mathrm{A}}} e^{-S^{b c}} \prod_{r=1}^{N} c \bar{c}\left(Z_{r}, \bar{Z}_{r}\right) \prod_{K=1}^{6 g-6+2 N}\left[\int d z \wedge d \bar{z} i\left(\mu_{K} b+\bar{\mu}_{K} \bar{b}\right)\right] .
\end{aligned}
$$

Here $S^{b c}$ is the action for the reparametrization ghosts, $\mu_{K}(K=1, \ldots, 6 g-6+2 N)$ denote the Beltrami differentials for the moduli parameters $T, \alpha, \theta$, and const. indicates a constant independent of the moduli parameters. The antighost insertion $\int d z \wedge d \bar{z} i\left(\mu_{K} b+\bar{\mu}_{K} \bar{b}\right)$ corresponding to the variations of $T, \alpha, \theta$ are given by the following contour integrals:

- The stretch corresponds to the variation $T \rightarrow T+\delta T$ of the height $T$ of cylinders. Let us order the interaction points $z_{I}(I=1, \ldots, 2 g-2+N)$ so that

$$
\operatorname{Re} \rho\left(z_{1}\right) \leq \operatorname{Re} \rho\left(z_{2}\right) \leq \cdots \leq \operatorname{Re} \rho\left(z_{2 g-2+N}\right)
$$




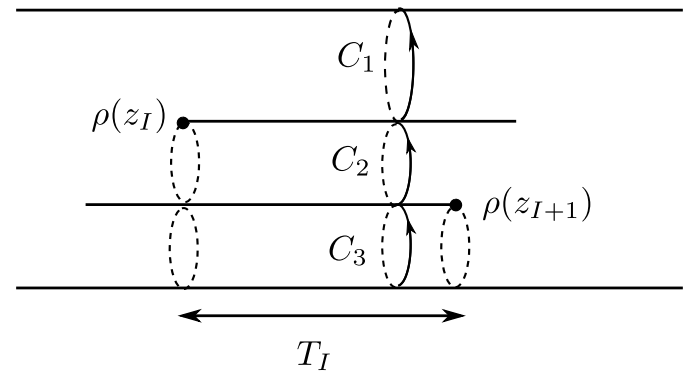

Figure 5. $C_{1}, C_{2}, C_{3}$ are the contours corresponding to the variation $T_{I} \rightarrow T_{I}+\delta T_{I}$.

and define the moduli parameters corresponding to the heights as

$$
T_{I} \equiv \operatorname{Re} \rho\left(z_{I+1}\right)-\operatorname{Re} \rho\left(z_{I}\right) \quad(I=1, \ldots, 2 g-3+N) .
$$

The antighost insertion corresponding to the deformation $T_{I} \rightarrow T_{I}+\delta T_{I}$ is given by

$$
\sum_{i} \oint_{C_{i}} d(\operatorname{Im} \rho)\left(b_{\rho \rho}+b_{\bar{\rho} \bar{\rho}}\right)=-i \sum_{i}\left(\oint_{C_{i}} \frac{d z}{\partial \rho} b_{z z}-\oint_{\bar{C}_{i}} \frac{d \bar{z}}{\bar{\partial} \bar{\rho}} b_{\bar{z} \bar{z}}\right)
$$

where $C_{i}$ denotes the contour around a cylinder which includes the region $\operatorname{Re} \rho\left(z_{I}\right) \leq$ $\operatorname{Re} \rho \leq \operatorname{Re} \rho\left(z_{I+1}\right)$ and the sum should be taken over all such contours. (See figure 5.) There are $2 g-3+N$ insertions of this kind.

- The twist $\theta \rightarrow \theta+\delta \theta$ corresponds to the rotation of one end of a cylinder with respect to the other. The antighost insertion should be

$$
\oint_{C_{\text {twist }}} d(\operatorname{Im} \rho)\left(b_{\rho \rho}-b_{\bar{\rho} \bar{\rho}}\right)=-i\left(\oint_{C_{\mathrm{twist}}} \frac{d z}{\partial \rho} b_{z z}+\oint_{\bar{C}_{\mathrm{twist}}} \frac{d \bar{z}}{\bar{\partial} \bar{\rho}} b_{\bar{z} \bar{z}}\right),
$$

where $C_{\text {twist }}$ is the contour around the cylinder which is twisted. There are $3 g-3+N$ insertions of this kind.

- The shift corresponds to the variation of the loop momenta preserving the momenta of the external lines. The antighost insertion for such a variation becomes

$$
-i\left(\oint_{C_{\text {shift }}} \frac{d z}{\partial \rho} b_{z z}+\oint_{\bar{C}_{\text {shift }}} \frac{d \bar{z}}{\bar{\partial} \bar{\rho}} b_{\bar{z} \bar{z}}\right),
$$

where $C_{\text {shift }}$ is taken as in figure 6 . There are $g$ insertions of this kind.

Therefore (C.29) can be expressed in terms of the contour integrals as

$$
\begin{aligned}
& \prod_{r=1}^{N}\left(\alpha_{r} e^{2 \operatorname{Re} \bar{N}_{00}^{r r}}\right) e^{-\Gamma\left[g_{z \bar{z}}^{\mathrm{A}}, \rho+\bar{\rho}\right]}\left(Z^{X}\left[g_{z \bar{z}}^{\mathrm{A}}\right]\right)^{-2} \\
& =\text { const. } \int[d b d \bar{b} d c d \bar{c}]_{g_{z \bar{z}}^{\mathrm{A}}} e^{-S^{b c}} \prod_{r=1}^{N} c \bar{c}\left(Z_{r}, \bar{Z}_{r}\right) \prod_{K=1}^{6 g-6+2 N}\left[\oint_{C_{K}} \frac{d z}{\partial \rho} b_{z z}+\varepsilon_{K} \oint_{\bar{C}_{K}} \frac{d \bar{z}}{\bar{\partial} \bar{\rho}} b_{\bar{z} \bar{z}}\right] .
\end{aligned}
$$

Here $\varepsilon_{K}=-1$ for the stretches and $\varepsilon_{K}=1$ for the twists and shifts. 


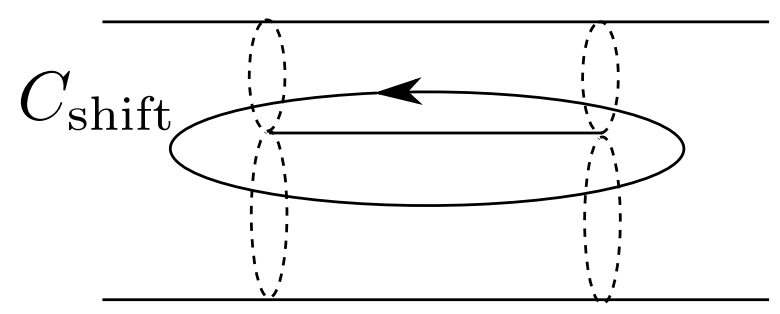

Figure 6. $C_{\text {shift }}$ corresponds to the variation of the momenta $p^{+}$flowing along it.

\section{A proof of (3.9)}

We would like to prove (3.9) by showing that the right hand side of (3.9) is equal to that of (3.8). We consider the generic situation in which $z_{I}, Z_{r}$ are all distinct.

What we do first is to rewrite the PCO's $X\left(z_{I}\right), \bar{X}\left(\bar{z}_{I}\right)$ using the existence of nilpotent fermionic charge

$$
\begin{aligned}
\hat{Q} \equiv & \oint \frac{d z}{2 \pi i} \partial \rho(z)\left[c\left(i \partial X^{+}-\frac{1}{2} \partial \rho\right)(z)+\frac{1}{2} \gamma \psi^{+}(z)\right] \\
& +\oint \frac{d \bar{z}}{2 \pi i} \bar{\partial} \bar{\rho}(\bar{z})\left[\bar{c}\left(i \bar{\partial} X^{+}-\frac{1}{2} \bar{\partial} \bar{\rho}\right)(\bar{z})+\frac{1}{2} \bar{\gamma} \bar{\psi}^{+}(\bar{z})\right] .
\end{aligned}
$$

One can show that $X\left(z_{I}\right)$ can be expressed as

$$
\begin{aligned}
X\left(z_{I}\right)=- & e^{\phi} T_{F}^{\mathrm{LC}}\left(z_{I}\right)+\left\{\hat{Q}, \oint_{z_{I}} \frac{d w}{2 \pi i} \frac{1}{w-z_{I}} \mathcal{O}(w) e^{\phi}\left(z_{I}\right)\right\} \\
& +\frac{1}{4} \oint_{z_{I}} \frac{d w}{2 \pi i} \frac{1}{w-z_{I}} \partial \rho \psi^{-}(w) e^{\phi}\left(z_{I}\right)+\frac{1}{4} b\left(\partial \eta e^{2 \phi}+\frac{1}{2} \eta \partial e^{2 \phi}\right)\left(z_{I}\right),
\end{aligned}
$$

where

$$
\begin{aligned}
\mathcal{O}=\frac{i}{\partial \rho} \partial X^{-} \beta+ & \frac{1}{2} \frac{b}{\partial \rho} \psi^{-} \\
+2 Q^{2} i\left[\left(\frac{5}{4} \frac{\left(\partial^{2} X^{+}\right)^{2}}{\left(\partial X^{+}\right)^{3}}-\frac{1}{2} \frac{\partial^{3} X^{+}}{\left(\partial X^{+}\right)^{2}}\right) \frac{2 \beta}{\partial \rho}\right. & \\
& \left.-\frac{2 \partial^{2} X^{+}}{\left(\partial X^{+}\right)^{2}} \partial\left(\frac{2 \beta}{\partial \rho}\right)+\frac{\partial^{2}\left(\frac{2 \beta}{\partial \rho}\right)}{\partial X^{+}}-\frac{\frac{2 \beta}{\partial \rho} \partial \psi^{+} \partial^{2} \psi^{+}}{2\left(\partial X^{+}\right)^{3}}\right],
\end{aligned}
$$

and $\hat{Q}$ satisfies the following identities:

$$
\begin{aligned}
& {\left[\hat{Q}, c \bar{c} e^{-\phi-\bar{\phi}} V_{r}^{\mathrm{DDF}}\left(Z_{r}, \bar{Z}_{r}\right)\right]=0,} \\
& {\left[\hat{Q}, e^{\phi} T_{F}^{\mathrm{LC}}\left(z_{I}\right)\right]=\left[Q, e^{\bar{\phi}} \bar{T}_{F}^{\mathrm{LC}}\left(\bar{z}_{I}\right)\right]=0,} \\
& {\left[\hat{Q}, \oint_{z_{I}} \frac{d w}{2 \pi i} \frac{1}{w-z_{I}} \partial \rho \psi^{-}(w) e^{\phi}\left(z_{I}\right)\right]=\left[\hat{Q}, \oint_{\bar{z}_{I}} \frac{d \bar{w}}{2 \pi i} \frac{1}{\bar{w}-\bar{z}_{I}} \bar{\partial} \bar{\rho} \bar{\psi}^{-}(\bar{w}) e^{\bar{\phi}}\left(\bar{z}_{I}\right)\right]=0,} \\
& {\left[\hat{Q}, \frac{1}{4} b\left(\partial \eta e^{2 \phi}+\frac{1}{2} \eta \partial e^{2 \phi}\right)\left(z_{I}\right)\right]=\left[\hat{Q}, \frac{1}{4} \bar{b}\left(\bar{\partial} \bar{\eta} e^{2 \bar{\phi}}+\frac{1}{2} \bar{\eta} \bar{\partial} e^{2 \bar{\phi}}\right)\left(\bar{z}_{I}\right)\right]=0,}
\end{aligned}
$$




$$
\begin{aligned}
& \left\{\hat{Q}, \mathcal{S}\left(\mathbf{z}, Z_{r}\right)\right\}=\left\{\hat{Q}, \overline{\mathcal{S}}\left(\overline{\mathbf{z}}, \bar{Z}_{r}\right)\right\}=0, \\
& \left\{\hat{Q}, e^{\phi} \partial\left(\psi^{+} e^{-\frac{Q^{2}}{2} \frac{i}{p_{r}^{+}} X_{L}^{+}}\right)\left(z_{I}\right)\right\}=\left\{\hat{Q}, e^{\bar{\phi}} \partial\left(\bar{\psi}^{+} e^{-\frac{Q^{2}}{2} \frac{i}{p_{r^{\prime}}^{+}} X_{R}^{+}}\right)\left(\bar{z}_{I}\right)\right\}=0 .
\end{aligned}
$$

The antighost insertions $\prod_{K=1}^{6 g-6+2 N}\left[\oint_{C_{K}} \frac{d z}{\partial \rho} b_{z z}+\varepsilon_{K} \oint_{\bar{C}_{K}} \frac{d \bar{z}}{\bar{\partial} \bar{\rho}} b_{\bar{z} \bar{z}}\right]$ is a product of the contour integrals of the types (C.32), (C.33) and (C.34). The anticommutator of $\hat{Q}$ with the contour integral of the type (C.32) becomes

$$
\begin{aligned}
& \left\{\hat{Q},-i \sum_{i}\left(\oint_{C_{i}} \frac{d z}{\partial \rho} b_{z z}-\oint_{\bar{C}_{i}} \frac{d \bar{z}}{\bar{\partial} \bar{\rho}} b_{\bar{z} \bar{z}}\right)\right\} \\
& \quad=-i \sum_{i}\left(\oint_{C_{i}} d z\left(i \partial X^{+}-\frac{1}{2} \partial \rho\right)-\oint_{\bar{C}_{i}} d \bar{z}\left(i \bar{\partial} X^{+}-\frac{1}{2} \bar{\partial} \rho\right)\right) .
\end{aligned}
$$

Since

$$
-i \sum_{i}\left(\oint_{C_{i}} d z i \partial X^{+}-\oint_{\bar{C}_{i}} d \bar{z} i \bar{\partial} X^{+}\right)
$$

and

$$
-i \sum_{i}\left(\oint_{C_{i}} d z \frac{1}{2} \partial \rho-\oint_{\bar{C}_{i}} d \bar{z} \frac{1}{2} \bar{\partial} \rho\right)
$$

are both equal to the total momentum in the + direction through the channel which is fixed by the external momenta, the right hand side of (D.5) should vanish. In the case of the contour integrals (C.33), (C.34), we obtain

$$
\begin{aligned}
& \left\{\hat{Q},-i\left(\oint_{C} \frac{d z}{\partial \rho} b_{z z}+\oint_{\bar{C}} \frac{d \bar{z}}{\bar{\partial} \bar{\rho}} b_{\bar{z} \bar{z}}\right)\right\} \\
& \quad=-i\left(\oint_{C} d z\left(i \partial X^{+}-\frac{1}{2} \partial \rho\right)+\oint_{\bar{C}} d \bar{z}\left(i \bar{\partial} X^{+}-\frac{1}{2} \bar{\partial} \rho\right)\right),
\end{aligned}
$$

which vanishes because $X^{+}$and $\rho+\bar{\rho}$ should be singlevalued. Hence $\hat{Q}$ commutes or anticommutes with all the quantities in (3.11). Therefore the second term on the right hand side of (D.2) does not contribute to the correlation functions, because it is $\hat{Q}$ exact. We can replace all the $X\left(z_{I}\right)$ in the correlation functions by

$$
-e^{\phi} T_{F}^{\mathrm{LC}}\left(z_{I}\right)+\frac{1}{4} \oint_{z_{I}} \frac{d w}{2 \pi i} \frac{1}{w-z_{I}} \partial \rho \psi^{-}(w) e^{\phi}\left(z_{I}\right)+\frac{1}{4} b\left(\partial \eta e^{2 \phi}+\frac{1}{2} \eta \partial e^{2 \phi}\right)\left(z_{I}\right),
$$

and similarly for $\bar{X}\left(\bar{z}_{I}\right)$. Then the third term in (D.7) can be omitted because of the ghost number conservation and similarly for the antiholomorphic sector.

Replacing $X\left(z_{I}\right)$ by

$$
-e^{\phi} T_{F}^{\mathrm{LC}}\left(z_{I}\right)+\frac{1}{4} \oint_{z_{I}} \frac{d w}{2 \pi i} \frac{1}{w-z_{I}} \partial \rho \psi^{-}(w) e^{\phi}\left(z_{I}\right)
$$


and similarly for $\bar{X}\left(\bar{z}_{I}\right)$, the right hand side of (3.9) becomes a sum of the right hand side of (3.8) and the terms which involve

$$
\oint_{z_{I}} \frac{d w}{2 \pi i} \frac{1}{w-z_{I}} \partial \rho \psi^{-}(w) e^{\phi}\left(z_{I}\right), \quad \oint_{\bar{z}_{I}} \frac{d \bar{w}}{2 \pi i} \frac{1}{\bar{w}-\bar{z}_{I}} \bar{\partial} \bar{\rho} \bar{\psi}^{-}(\bar{w}) e^{\bar{\phi}}\left(\bar{z}_{I}\right)
$$

or

$$
e^{\phi} \partial\left(\psi^{+} e^{-\frac{Q^{2}}{2} \frac{i}{p_{r}^{+}} X_{L}^{+}}\right)\left(z_{I}\right), \quad e^{\bar{\phi}} \partial\left(\bar{\psi}^{+} e^{-\frac{Q^{2}}{2} \frac{i}{p_{r^{\prime}}^{+}} X_{R}^{+}}\right)\left(\bar{z}_{I}\right),
$$

which appear in (3.13) and (3.14), in place of $e^{\phi} T_{F}^{\mathrm{LC}}\left(z_{I}\right), e^{\bar{\phi}} \bar{T}_{F}^{\mathrm{LC}}\left(\bar{z}_{I}\right)$. The $X^{ \pm}$CFT part of such terms are of the form

$$
\begin{gathered}
\left\langle\mathcal{O}^{-}\left(z_{I_{1}}\right) \cdots \mathcal{O}^{-}\left(z_{I_{n}}\right) \mathcal{O}^{+}\left(z_{I_{n+1}}\right) \cdots \mathcal{O}^{+}\left(z_{I_{n+m}}\right) \overline{\mathcal{O}}^{-} \ldots \overline{\mathcal{O}}^{+} \ldots\right. \\
\left.\quad \times\left(\text { contributions from } \mathcal{S}, \overline{\mathcal{S}}, V_{r}^{\mathrm{DDF}}\right)\right\rangle^{X^{ \pm}}
\end{gathered}
$$

where

$$
\begin{aligned}
\mathcal{O}^{-}\left(z_{I}\right) & \equiv \oint_{z_{I}} \frac{d w}{2 \pi i} \frac{1}{w-z_{I}} \partial \rho \psi^{-}(w), \\
\mathcal{O}^{+}\left(z_{I}\right) & \equiv \partial\left(\psi^{+} e^{-\frac{Q^{2}}{2} \frac{i}{p_{r}^{+}} X_{L}^{+}}\right)\left(z_{I}\right),
\end{aligned}
$$

and the antiholomorphic versions are defined in a similar way. Here $z_{I_{1}}, \ldots, z_{I_{n+m}}$ are all distinct. We would like to show that the correlation functions of the form (D.11) vanish. (B.21) implies that in calculating correlation functions of the form (D.11), all the $\psi^{+}$'s in $\mathcal{O}^{+}$'s should be contracted with $\psi^{-}$'s, which come from $\mathcal{O}^{-}\left(z_{I}\right)$. Therefore (D.11) with $m \neq 0$ should involve a factor of the form

$$
\oint_{z_{I}} \frac{d w}{2 \pi i} \frac{1}{w-z_{I}} \partial \rho \psi^{-}(w) \partial^{k} \psi^{+}\left(z_{J}\right)
$$

for some integer $k \geq 0$, with $z_{I} \neq z_{J}$. (D.13) vanishes because the contraction does not have any poles at $w=z_{I}$ and $\partial \rho\left(z_{I}\right)=0$. Therefore the correlation function (D.11) vanishes if it involves $\mathcal{O}^{+}$or $\overline{\mathcal{O}}^{+}$. What remains to be done is to prove the correlation functions of the form

$$
\left\langle\mathcal{O}^{-}\left(z_{I_{1}}\right) \cdots \mathcal{O}^{-}\left(z_{I_{n}}\right) \overline{\mathcal{O}}^{-} \cdots \times\left(\text { contributions from } \mathcal{S}, \overline{\mathcal{S}}, V_{r}^{\mathrm{DDF}}\right)\right\rangle^{X^{ \pm}}
$$

vanish using (B.21). The contractions of $\psi^{-}$'s in $\mathcal{O}^{-}$with $\psi^{+}$'s from $V_{r}^{\text {DDF }}$ do not contribute because

$$
\oint_{z_{I}} \frac{d w}{2 \pi i} \frac{1}{w-z_{I}} \partial \rho \psi^{-}(w) \partial^{k} \psi^{+}\left(Z_{r}\right)=0 .
$$

The contractions of $\psi^{-}$'s in $\mathcal{O}^{-}$and $\psi^{+}$'s from $\mathcal{S}$ inevitably induce a factor of the form

$$
\oint_{z_{I}} \frac{d w}{2 \pi i} \frac{1}{w-z_{I}} \partial \rho \psi^{-}(w) \partial^{k} \psi^{+}(z)=0,
$$




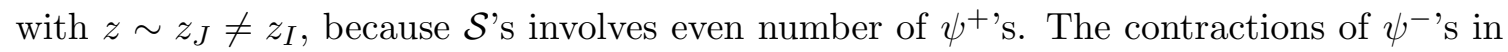
$\mathcal{O}^{-}$and $\psi^{+}$'s from $S_{\text {int }}$ necessarily induce a factor of the form

$$
\oint_{z_{I}} \frac{d w}{2 \pi i} \frac{1}{w-z_{I}} \partial \rho \psi^{-}(w) \partial^{k} \psi^{+}\left(z_{J}\right)=0
$$

with $z_{J} \neq z_{I}$, because $S_{\text {int }}$ is localized at the interaction points and involves even number of $\psi^{+}$'s.

Thus we have shown that the terms of the form (D.11) all vanish and the right hand side of (3.9) is equal to that of (3.8).

Open Access. This article is distributed under the terms of the Creative Commons Attribution License (CC-BY 4.0), which permits any use, distribution and reproduction in any medium, provided the original author(s) and source are credited.

\section{References}

[1] S. Mandelstam, Interacting String Picture of the Neveu-Schwarz-Ramond Model, Nucl. Phys. B 69 (1974) 77 [INSPIRE].

[2] S. Mandelstam, Interacting String Picture of the Fermionic String, Prog. Theor. Phys. Suppl. 86 (1986) 163 [INSPIRE].

[3] S.-J. Sin, Geometry of Super Light Cone Diagrams and Lorentz Invariance of Light Cone String Field Theory. 2. Closed Neveu-Schwarz String, Nucl. Phys. B 313 (1989) 165 [INSPIRE].

[4] M.B. Green and J.H. Schwarz, Superstring Interactions, Nucl. Phys. B 218 (1983) 43 [INSPIRE].

[5] M.B. Green, J.H. Schwarz and L. Brink, Superfield Theory of Type II Superstrings, Nucl. Phys. B 219 (1983) 437 [INSPIRE].

[6] D.J. Gross and V. Periwal, Heterotic String Light Cone Field Theory, Nucl. Phys. B 287 (1987) 1 [INSPIRE].

[7] Y. Baba, N. Ishibashi and K. Murakami, Light-Cone Gauge Superstring Field Theory and Dimensional Regularization, JHEP 10 (2009) 035 [arXiv:0906.3577] [INSPIRE].

[8] Y. Baba, N. Ishibashi and K. Murakami, Light-Cone Gauge String Field Theory in Noncritical Dimensions, JHEP 12 (2009) 010 [arXiv:0909.4675] [INSPIRE].

[9] Y. Baba, N. Ishibashi and K. Murakami, Light-cone Gauge NSR Strings in Noncritical Dimensions, JHEP 01 (2010) 119 [arXiv:0911.3704] [INSPIRE].

[10] Y. Baba, N. Ishibashi and K. Murakami, Light-cone Gauge Superstring Field Theory and Dimensional Regularization II, JHEP 08 (2010) 102 [arXiv:0912.4811] [INSPIRE].

[11] N. Ishibashi and K. Murakami, Light-cone Gauge NSR Strings in Noncritical Dimensions II - Ramond Sector, JHEP 01 (2011) 008 [arXiv: 1011.0112] [INSPIRE].

[12] N. Ishibashi and K. Murakami, Spacetime Fermions in Light-cone Gauge Superstring Field Theory and Dimensional Regularization, JHEP 07 (2011) 090 [arXiv:1103.2220] [INSPIRE].

[13] N. Ishibashi and K. Murakami, Multiloop Amplitudes of Light-cone Gauge Bosonic String Field Theory in Noncritical Dimensions, JHEP 09 (2013) 053 [arXiv:1307.6001] [INSPIRE]. 
[14] N. Ishibashi and K. Murakami, Worldsheet theory of light-cone gauge noncritical strings on higher genus Riemann surfaces, JHEP 06 (2016) 087 [arXiv: 1603.08337] [INSPIRE].

[15] J. Greensite and F.R. Klinkhamer, New Interactions for Superstrings, Nucl. Phys. B 281 (1987) 269 [INSPIRE].

[16] J. Greensite and F.R. Klinkhamer, Contact Interactions in Closed Superstring Field Theory, Nucl. Phys. B 291 (1987) 557 [inSPIRE].

[17] J. Greensite and F.R. Klinkhamer, Superstring Amplitudes and Contact Interactions, Nucl. Phys. B 304 (1988) 108 [inSPIRE].

[18] M.B. Green and N. Seiberg, Contact Interactions in Superstring Theory, Nucl. Phys. B 299 (1988) 559 [INSPIRE].

[19] C. Wendt, Scattering Amplitudes and Contact Interactions in Witten's Superstring Field Theory, Nucl. Phys. B 314 (1989) 209 [InSPIRE].

[20] N. Ishibashi, Light-cone gauge superstring field theory in linear dilaton background, arXiv: 1605.04666 [INSPIRE].

[21] A. Sen, BV Master Action for Heterotic and Type II String Field Theories, JHEP 02 (2016) 087 [arXiv: 1508.05387] [INSPIRE].

[22] A. Sen, Unitarity of Superstring Field Theory, JHEP 12 (2016) 115 [arXiv:1607.08244] [INSPIRE].

[23] A. Sen, One Loop Mass Renormalization of Unstable Particles in Superstring Theory, JHEP 11 (2016) 050 [arXiv:1607.06500] [INSPIRE].

[24] A. Sen, Off-shell Amplitudes in Superstring Theory, Fortsch. Phys. 63 (2015) 149 [arXiv: 1408.0571] [INSPIRE].

[25] A. Sen, Gauge Invariant 1PI Effective Action for Superstring Field Theory, JHEP 06 (2015) 022 [arXiv: 1411.7478] [INSPIRE].

[26] A. Sen and E. Witten, Filling the gaps with PCO's, JHEP 09 (2015) 004 [arXiv: 1504.00609] [INSPIRE].

[27] S. Arakelov, Intersection Theory of Divisors on an Arithmetic Surface, Math. USSR Izv. 8 (1974) 1167.

[28] E. D'Hoker and S.B. Giddings, Unitary of the Closed Bosonic Polyakov String, Nucl. Phys. B 291 (1987) 90 [INSPIRE].

[29] K. Aoki, E. D'Hoker and D.H. Phong, Unitarity of Closed Superstring Perturbation Theory, Nucl. Phys. B 342 (1990) 149 [InSPIRE].

[30] E. D'Hoker and D.H. Phong, The Geometry of String Perturbation Theory, Rev. Mod. Phys. 60 (1988) 917 [INSPIRE].

[31] S. Mandelstam, The Interacting String Picture and Functional Integration, lectures given at the Workshop on Unified String Theory, Santa Babara, CA U.S.A., Jul 29 - Aug 161985.

[32] L. Álvarez-Gaumé, J.B. Bost, G.W. Moore, P.C. Nelson and C. Vafa, Bosonization on Higher Genus Riemann Surfaces, Commun. Math. Phys. 112 (1987) 503 [InSPIRE].

[33] E.P. Verlinde and H.L. Verlinde, Chiral Bosonization, Determinants and the String Partition Function, Nucl. Phys. B 288 (1987) 357 [INSPIRE]. 
[34] M.J. Dugan and H. Sonoda, Functional Determinants on Riemann Surfaces, Nucl. Phys. B 289 (1987) 227 [INSPIRE].

[35] H. Sonoda, Conformal Field Theories With First Order Lagrangians, Phys. Lett. B 197 (1987) 167 [INSPIRE].

[36] J.J. Atick and A. Sen, Spin Field Correlators on an Arbitrary Genus Riemann Surface and Nonrenormalization Theorems in String Theories, Phys. Lett. B 186 (1987) 339 [INSPIRE].

[37] H. Sonoda, Functional Determinants on Punctured Riemann Surfaces and Their Application to String Theory, Nucl. Phys. B 294 (1987) 157 [INSPIRE].

[38] E. D'Hoker and D.H. Phong, Functional Determinants on Mandelstam Diagrams, Commun. Math. Phys. 124 (1989) 629 [inSPIRE]. 